\title{
Nonlinear Transverse Vibrations of a Slightly Curved Beam resting on Multiple Springs
}

\author{
E. Özkaya, M. Sarıüll and H. Boyacı \\ Department of Mechanical Engineering, Celal Bayar University, Muradiye, 45140 Manisa, Turkey
}

\begin{abstract}
(Received 9 June 2014; accepted 11 May 2015)
In this study, nonlinear vibrations of a slightly curved beam of arbitrary rise functions is handled in case it rests on multiple springs. The beam is simply supported on both ends and is restricted in longitudinal directions using the supports. Thus, the equations of motion have nonlinearities due to elongations during vibrations. The method of multiple scales (MMS), a perturbation technique, is used to solve the integro-differential equation analytically. Primary and 3 to 1 internal resonance cases are taken into account during steady-state vibrations. Assuming the rise functions are sinusoidal in numerical analysis, the natural frequencies are calculated exactly for different spring numbers, spring coefficients, and spring locations. Frequency-amplitude graphs and frequency-response graphs are plotted by using amplitude-phase modulation equations.
\end{abstract}

\section{INTRODUCTION}

Some beam elements of bridges, rails, and automotive industries are designed for the purpose of preventing impact by modern engineers. One of these elements is a curved beam model resting on an elastic foundation. Matter considered in these models, which have nonlinear behavior, is the resonance case of the system. If the system comes into a resonance state, the amplitudes increase dangerously, which is an unwanted case. Nonlinear problems of the model must be examined in order to prevent these cases that may occur at any time during vibration. For this reason, the linear part of the system must first be solved analytically and then the effects of nonlinearity should be added to the solutions. Thus, nonlinear vibrations of the system can be investigated. Before introducing the background of curved or pre-buckled beams, some studies related to our investigation must be mentined. Nayfeh and Mook reviewed and presented relevant works to the field up to 1979 in their book. ${ }^{1}$ Cha derived governing equations for a linear elastica carrying a number of lumped masses, springs, and viscous dampers. $^{2}$ Albarracn et al. studied free vibrations of a uniform beam with intermediate constraints and ends that were elastically restrained against rotation and translation. ${ }^{3}$ Wang and Qiao derived a general solution of the modal displacement of a beam with arbitrary discontinuities. ${ }^{4}$ Wiedemann studied an arbitrary system of Euler-Bernoulli beams that were interconnected by arbitrary joints and subject to arbitrary boundary conditions. ${ }^{5}$ Huang and Chen studied structures with multiple attachments that were subjected to axial forces and oscillations. ${ }^{6}$ Regarding some assumptions in their model, they examined the remaining model with the pure buckling problem, the free vibration problem, and the general eigenvalue problem. Kelly and Srinivas investigated elastically connected axially-loaded beams, which may be attached to a Winkler foundation. ${ }^{7}$ Wang et al. studied the nonlinear interaction of an inextensional beam on an elastic foundation with a three-toone internal resonance. ${ }^{8}$

In some studies, the beam was assumed to have a rising function so the curvature effect on vibrations of the beam could be investigated. Some of these studies were such that Rehfield derived the equations of motion of a shallow arch with an arbitrary rise function and studied the free vibrations approximately. ${ }^{9}$ Singh and Ali studied a moderately thick clamped beam with a sinusoidal rise function by adding the effects of transverse shears and rotary inertia. ${ }^{10}$ Hajianmaleki and Qatu focused on the last two decades of research (1989-2012) done on vibration analysis. ${ }^{11}$ They reviewed various beam theories such as thin (or classical), thick (or shear deformation), layerwise beam theories, and different methods for solving equations of motion, such as the transfer matrix method and the finite element method. Tien et al. studied the dynamics of a shallow arch subjected to harmonic excitation. ${ }^{12}$ In the presence of both external and 1:1 internal resonance, he examined the bifurcation behavior of the shallow arch system. Using two beam elements, one has three degree-of-freedom and other four. Krishnan and Suresh studied the static and free vibrations of curved beams. ${ }^{13} \mathrm{Oz}$ et al. examined a simply supported and slightly curved beam resting on an elastic foundation with cubic non-linearities. ${ }^{14}$ Considering freeundamped and forced-damped vibrations, they analyzed the effects of the elastic foundation, axial stretching, and curvature on the vibrations of the beams. Using a systematic theoretical procedure, Lin presented a static analysis of extensional circular-curved Timoshenko beams with general nonhomogeneous elastic boundary conditions and found the generalized Green function of differential equations. ${ }^{15}$ For a general state of non-uniform initial stress, Chen and Shen derived the virtual work expressions of initially stressed curved beams. ${ }^{16}$ They investigated the influence of arc segment angles, elastic foundations, and initial stresses on natural frequencies. Nayfeh et 
al. studied how to construct the nonlinear normal modes of a fixed-fixed buckled beam about its first post-buckling mode. ${ }^{17}$ Considering the cases of three-to-one and one-to-one internal resonances to solve the problem, they used the method of multiple scales. Tarnopolskaya et al. examined the vibrational behavior of beams with arbitrarily varying curvature and cross-section in the lower region of the spectrum. ${ }^{18}$ They examined whether or not the mode transition took place for a particular type of beam curvature and cross-section. Lestari and Hanagud found closed-form exact solutions to the problem of nonlinear vibrations of buckled beams. ${ }^{19}$ They assumed that their model consisted of axial springs in spite of it having general support conditions. Lacarbonara et al. developed the open-loop nonlinear control strategy and applied it to a hinged hinged shallow arch. ${ }^{20}$ They assumed the beam had been subjected to a longitudinal end-displacement with a frequency that was twice the frequency of the second mode (principal parametric resonance). Lacarbonara and Rega studied general conditions for orthogonality of the non-linear normal modes of one-dimensional systems with arbitrary linear, quadratic, and cubic non-linearities. ${ }^{21}$ Considering the cases of two-to-one, three-to-one, and one-to-one internal resonances in a class of shallow symmetric structural systems, they examined undamped and unforced vibrations. Wu and Chiang presented a simple, straightforward, and systematic technique to derive the displacement functions for the radial, or normal, tangential and rotational displacements of an arch element. ${ }^{22}$ In their study, static equilibrium equations were investigated further. Adessi et al. studied the regime of high pre-stressed beams. ${ }^{23}$ They examined post-buckling configurations of the beam considering a lumped mass that is rigidly clamped to the beam at an arbitrary point along its span and assuming different boundary conditions (simply supported and hingedhinged). Lacarbonara et al. investigated the non-linear oneto-one interactions excited by an external primary-resonance base acceleration of a hinged-hinged imperfect beam with a torsional spring at one end and possessing veering between the frequencies of the lowest two modes. ${ }^{24}$ Ecsedi and Dluhi studied a non-homogeneous curved beam formulated in cylindrical coordinates and examined the static and dynamic analysis of the curved beam. ${ }^{25}$ Lee et al. studied how to derive the equations of motion for a clampedclamped curved beam subjected to transverse sinusoidal loads. By using the assumed mode, the Galerkin method, and assuming a single mode approach, they determined the effect of parametric excitation near the symmetric mode resonance frequency. ${ }^{26} \mathrm{Oz}$ and Das investigated natural frequencies of a circular curved beam with a Mode 1 open transverse crack by using FEM. ${ }^{27} \mathrm{Oz}$ and Pakdemirli studied vibrations of simply supported shallow curved beams. ${ }^{28}$ Assuming the curvature of the beam had sinusoidal and parabolic functions, they searched whether or not there were 2:1 internal resonances. By assuming the sinusoidal rising function for the initial curvature of the beam, Erdogan et al. studied nonlinear vibrations of curved beams carrying a concentrated mass and multiple concentrated masses. ${ }^{29,30} \mathrm{Xi}$ uchang et al. proposed a wave approach to investigate the wave

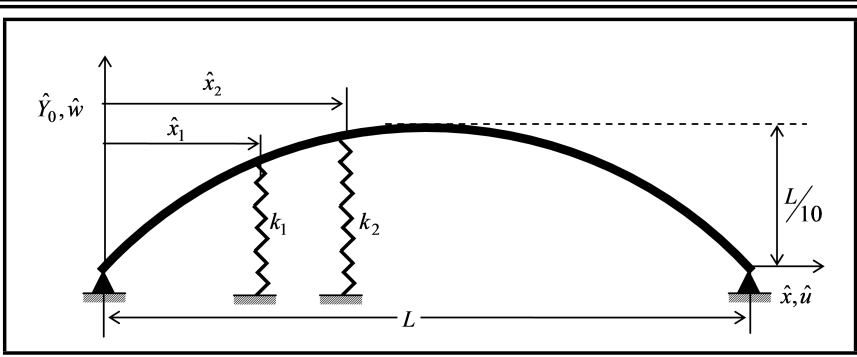

Figure 1. A curved beam resting on multiple springs.

propagation in the structural waveguides with curved beam components. ${ }^{31}$

In the recent years, some researchers focused on the continua resting on partially supported elastic foundation/multiple springs. These studies were such that Stncioiu et al. studied the dynamics of a two-axle system travelling along a continuous elastic beam resting on elastic supports modeled as linear springs. ${ }^{32}$ During its travel along the vibrating beam, effects resulting from the presence of intermediate elastic supports were examined. Motaghian et al. proposed an exact solution to the free vibration problem of beams having mixed boundary conditions. ${ }^{33}$ By using the Fourier series, they solved governing differential equations of beams that had underlying elastic springs, which occupied a particular length of the beam. Motaghian et al. proposed an exact solution to the free vibration problem of plates having mixed boundary conditions. ${ }^{34}$ By using the Fourier series, they solved governing differential equations of plates that have underlying elastic springs which occupy an arbitrary area of the plate. Ghayesh investigated the free and forced vibrations of a Kelving-Voigt viscoelastic beam supported by a nonlinear spring. ${ }^{35}$ Linear and nonlinear frequencies of the system were analyzed by considering the nonlinear spring effect. Sari and Pakdemirli studied the dynamic behavior of a slightly curved microbeam that had nonideal boundary conditions. ${ }^{36}$ They also presented references for the choice of resonable resonant conditions, design applications, and industrial applications of such systems.

In this work, nonlinear vibrations of a curved beam resting on multiple springs were investigated. The beam was assumed to have an arbitrarily curvature function and simply supported at both ends. To seek an analytical solution to the problem, the method of multiple scales (MMS), a perturbation method, was used. Primary and 3:1 internal resonance cases were studied in detail. Assuming the curvature of the beam was a sinusoidal function, the numerical solutions were obtained for steady-state phase of vibrations.

\section{FORMULATION OF THE PROBLEM}

In Fig. 1, the curved beam-spring system is restricted on both ends with immovable supports. In such a system, $\hat{w}_{m}$ and $\hat{u}_{m}$ denote transversal and longitudinal displacements respectively. Assuming that the ratio of the beam's maximum amplitude to its projected lenght $L$ is equal to $1 / 10$, let us keep in mind that the beam's curvature function is in the $\hat{Y}_{0}$ arising function. Let us assume that $n$ number of springs is attached 
under the beam, $k_{m}$ is the spring coefficient, and $\hat{x}_{m}$ is the distance of these springs from the immovable end at the left-hand side. In order to analyize the equations of motion within this system, we used its potential $(U)$ and kinetic $(T)$ energies as defined below:

$$
\begin{gathered}
U= \\
\frac{1}{2} \sum_{m=0}^{n} E \cdot A \cdot \int_{\hat{x}_{m}}^{\hat{x}_{m+1}}\left(\hat{u}_{m+1}^{\prime}+\hat{Y}_{0}^{\prime} \cdot \hat{w}_{m+1}^{\prime}+\frac{1}{2} \cdot \hat{w}_{m+1}^{\prime 2}\right)^{2} d \hat{x} \\
\quad+\frac{1}{2} \cdot \sum_{m=0}^{n} E \cdot I \cdot \int_{\hat{x}_{m}}^{\hat{x}_{m+1}} \hat{w}_{m+1}^{\prime \prime 2} d \hat{x} \\
+\left.\frac{1}{2} \cdot \sum_{m=0}^{n} k_{m+1} \cdot \hat{w}_{m+1}^{2}\right|_{x_{m+1}=\hat{x}_{m+1}, t=\hat{t}} ; \quad(1) \\
T=\frac{1}{2} \sum_{m=0}^{n} \rho \cdot A \cdot \int_{\hat{x}_{m}}^{\hat{x}_{m+1}} \dot{\hat{w}}_{m+1}^{2} d \hat{x}, \quad \\
\hat{x}_{0}=0, \quad \hat{x}_{n+1}=L, \quad m=0, \quad 1 \ldots n . \quad \text { (2) }
\end{gathered}
$$

In Eqs. (1) and (2), $E$ is the young modulus, $\rho$ is the density, $A$ is the cross sectional area of the beam, and $I$ is the moment of inertia of the beam cross-section with respect to the neutral axis. (') and (') denote differentiations with respect to the time $t$ and the spatial variable $x$ respectively.

Inserting these energy terms defined Eqs. (1) and (2) into the Hamilton Principle formulation as shown below:

$$
\delta \int_{\hat{t}_{1}}^{\hat{t}_{2}}(T-U) d \hat{t}=0
$$

and by invoking the necessary calculations, longitudinal displacement term $\left(u_{m}\right)$ could be eliminated from the equations of motion in the tranverse direction. Thus, the equations of motion can be written as follows:

$$
\begin{array}{r}
\rho \cdot A \cdot \ddot{\hat{w}}_{m+1}+E \cdot I \cdot \hat{w}_{m+1}^{\imath v}= \\
\frac{E \cdot A}{L} \cdot\left[\sum_{r=0}^{n} \int_{\hat{x}_{r}}^{\hat{x}_{r+1}}\left\{\hat{Y}_{0}^{\prime} \cdot \hat{w}_{r+1}^{\prime}+\frac{1}{2} \cdot \hat{w}_{r+1}^{2}\right\} d \hat{x}\right] \\
\cdot\left(\hat{Y}_{0}^{\prime \prime}+\hat{w}_{m+1}^{\prime \prime}\right) .
\end{array}
$$

In Eq. (4), the equation of motion for the system consists of $n+1$ equations in number. The equations of the motion and boundary conditions were dependent on the size of the system and the materials used. In order to make them independent from the dimensional parameters, the following defi- nitions must be made:

$$
\begin{array}{r}
w_{p}=\hat{w}_{p} / L, \quad Y_{0}=\hat{Y}_{0} / L, \quad x=\hat{x} / L, \\
t=\sqrt{\frac{E \cdot I}{\rho \cdot A \cdot L^{2}}} \cdot \hat{t}, \quad I=r 2 \cdot A, \\
\eta_{p}=\hat{x}_{p} / L, \quad \tau_{p}=\frac{k_{p} \cdot L^{3}}{E \cdot I} ;
\end{array}
$$

where $r$ is the radius of gyration of the beam's cross section, $\tau_{m}$ is the stiffness ratio between the spring and the beam, and $\eta_{m}$ is the dimensionless distance of the spring from left handside support. Adding dimensionless damping $(\vec{\mu})$ and external forcing $(\vec{F})$ terms after non-dimensionalization, equations of motion via boundary and continuity conditions can be rewritten as follows:

$$
\begin{gathered}
\ddot{w}_{m+1}+w_{m+1}^{v v}+2 \cdot \vec{\mu} \cdot \dot{w}_{m+1}= \\
{\left[\sum_{r=0}^{n} \int_{\eta_{r}}^{\eta_{r+1}}\left\{Y_{0}^{\prime} \cdot w^{\prime}{ }_{r+1}+\frac{1}{2} \cdot w_{r+1}^{\prime 2}\right\} d x\right]} \\
\cdot\left(Y^{\prime \prime}{ }_{0}+w^{\prime \prime}{ }_{m+1}\right)+\vec{F}_{m+1} \cdot \cos (\Omega \cdot t) ;
\end{gathered}
$$

$$
\begin{gathered}
\left.w_{p}\right|_{x=\eta_{p}}=\left.w_{p+1}\right|_{x=\eta_{p}}, \\
\left.w^{\prime}\right|_{x=\eta_{p}}=\left.w_{p+1}^{\prime}\right|_{x=\eta_{p}}, \\
\left.w^{\prime \prime}\right|_{x=\eta_{p}}=\left.w^{\prime \prime}{ }_{p+1}\right|_{x=\eta_{p}}, \\
\left.\left(w^{\prime \prime \prime}{ }_{p}-w^{\prime \prime \prime}{ }_{p+1}\right)\right|_{x=\eta_{p}}=\left.\tau_{p} \cdot w_{p}\right|_{x=\eta_{p}} \\
\left.w_{1}\right|_{x=\eta_{0}}=\left.w^{\prime \prime}\right|_{x=\eta_{0}}=\left.w_{n+1}\right|_{x=\eta_{n+1}}=\left.w^{\prime \prime}{ }_{n+1}\right|_{x=\eta_{n+1}}=0 \\
\eta_{0}=0, \\
\eta_{n+1}=1
\end{gathered}
$$

where $\Omega$ is the frequency of the external forcing.

\section{ANALYTICAL SOLUTIONS}

\subsection{Multiple Scales Method - A Perturbation Method}

In this section, the method of multiple scales (MMS)(Nayfeh ${ }^{37,38}$ ) will be applied to the partial differential equations and corresponding boundary conditions directly in order to search approximate solutions to the problem. Eq. (6) is assumed to have an expansion solution as follows:

$$
w_{p+1}(x, t ; \varepsilon)=\sum_{j=1}^{3} \varepsilon^{j} \cdot w_{(p+1) j}\left(x, T_{0}, T_{1}, T_{2}\right) ;
$$

where $\epsilon$ is a small bookkeeping parameter artificially inserted into the equations, $T_{0}=t$ is the fast time scale, and $T_{1}=\varepsilon \cdot t$ and $T_{2}=\varepsilon_{2} \cdot t$ were the slow time scales in MMS. In order 
to counter the effects of the nonlinear terms via the same order of damping and forcing, the forcing and damping terms were ordered as follows:

$$
\vec{\mu}=\varepsilon^{2} \cdot \mu, \quad \vec{F}_{p+1}=\varepsilon^{3} \cdot F_{p+1} .
$$

Let us assume the curvature function of the beam as $Y_{0} \approx$ $O(1)$, which means that its order corresponds to first order of the general system. Under this assumption, inserting Eqs. (8) and (9) into Eq. (6) and separating the terms of each order, one finds the following equations: Order $\varepsilon(j=1)$ :

$D_{0}^{2} \cdot w_{(m+1) 1}+w_{(m+1) 1}^{\imath v}=\left\{\sum_{r=0}^{n} \int_{\eta_{r}}^{\eta_{r+1}} Y_{0}^{\prime} \cdot w^{\prime}{ }_{(r+1) 1} d x\right\} \cdot Y_{0}^{\prime \prime}$.

$\operatorname{Order} \varepsilon^{2}(j=2)$ :

$$
\begin{aligned}
D_{0}^{2} \cdot w_{(m+1) 2}+w_{(m+1) 2}^{\imath v}= & -2 \cdot D_{0} \cdot D_{1} \cdot w_{(m+1) 1} \\
+ & \left\{\sum_{r=0}^{n} \int_{\eta_{r}}^{\eta_{r+1}} Y_{0}^{\prime} \cdot w_{(r+1) 2}^{\prime} d x\right\} \cdot Y^{\prime \prime}{ }_{0} \\
& +\frac{1}{2} \cdot\left\{\sum_{r=0}^{n} \int_{\eta_{r}}^{\eta_{r+1}} w_{(r+1) 1}^{\prime 2} d x\right\} \cdot Y^{\prime \prime}{ }_{0} \\
+ & \left\{\sum_{r=0}^{n} \int_{\eta_{r}}^{\eta_{r+1}} Y_{0}^{\prime} \cdot w_{(r+1) 1}^{\prime} d x\right\} \cdot w^{\prime \prime}{ }_{(m+1) 1} .
\end{aligned}
$$

$\operatorname{Order} \varepsilon^{3}(j=3)$ :

$$
\begin{aligned}
D_{0}^{2} \cdot w_{(m+1) 3}+w_{(m+1) 3}^{\imath v}= & \\
-2 \cdot & \mu \cdot D_{0} \cdot w_{(m+1) 1}-2 \cdot D_{0} \cdot D_{1} \cdot w_{(m+1) 2} \\
-\left(D_{1}^{2}+\right. & \left.+2 \cdot D_{0} \cdot D_{2}\right) \cdot w_{(m+1) 1}+F_{m+1} \cdot \cos (\Omega \cdot t) \\
& +\left\{\sum_{r=0}^{n} \int_{\eta_{r}}^{\eta_{r+1}} Y_{0}^{\prime} \cdot w_{(r+1) 3}^{\prime} d x\right\} \cdot Y^{\prime \prime}{ }_{0} \\
+ & \left\{\sum_{r=0}^{n} \int_{\eta_{r}}^{\eta_{r+1}} w_{(r+1) 1}^{\prime} \cdot w_{(r+1) 2}^{\prime} d x\right\} \cdot Y^{\prime \prime}{ }_{0} \\
+ & \left\{\sum_{r=0}^{n} \int_{\eta_{r}}^{\eta_{r+1}} Y_{0}^{\prime} \cdot w_{(r+1) 2}^{\prime} d x\right\} \cdot w^{\prime \prime}{ }_{(r+1) 1} \\
+ & \frac{1}{2} \cdot\left\{\sum_{r=0}^{n} \int_{\eta_{r}}^{\eta_{r+1}} w_{(r+1) 1}^{\prime 2} d x\right\} \cdot w^{\prime \prime}{ }_{(p+1) 1} \\
+ & \left\{\sum_{r=0}^{n} \int_{\eta_{r}}^{\eta_{r+1}} Y_{0}^{\prime} \cdot w_{(r+1) 1}^{\prime} d x\right\} \cdot w^{\prime \prime}{ }_{(p+1) 2} ; \quad(12)
\end{aligned}
$$

where $D_{n} \equiv \partial / \partial T_{n}$ is the derivative with respect to time and given in Appendix (A.1). The conditions that were necessary for solving Eqs. (10) to (12), are given as below:

$$
\begin{gathered}
\left.w_{p j}\right|_{x=\eta_{p}}=\left.w_{(p+1) j}\right|_{x=\eta_{p}}, \\
\left.w_{p j}^{\prime}\right|_{x=\eta_{p}}=\left.w_{(p+1) j}^{\prime}\right|_{x=\eta_{p}}, \\
\left.w^{\prime \prime}{ }_{p j}\right|_{x=\eta_{p}}=\left.w^{\prime \prime}{ }_{(p+1) j}\right|_{x=\eta_{p}}, \\
\left.\left(w^{\prime \prime \prime \prime}{ }_{p j}-w^{\prime \prime \prime}{ }_{(p+1) j}\right)\right|_{x=\eta_{p}}=\left.\tau_{p} \cdot w_{p j}\right|_{x=\eta_{p}} \\
\left.w_{1 j}\right|_{x=\eta_{0}}=\left.w^{\prime \prime}{ }_{1 j}\right|_{x=\eta_{0}}=\left.w_{(n+1) j}\right|_{x=\eta_{n+1}}= \\
\left.w^{\prime \prime}{ }_{(n+1) j}\right|_{x=\eta_{n+1}}=0 \quad j=1,2,3 .
\end{gathered}
$$

Eq. (10) from order $\varepsilon_{1}$ corresponds to the linear problem of the system and other orders in Eqs. (11) and (12) to the nonlinear problem. These cases were investigated seperately when these equations were being solved. Firstly, solutions of the primary resonance case were searched. Secondly, 3:1 resonance case has been investigated by assuming there is three-to-one ratio between any two natural frequencies.

\subsection{Primary Resonance}

Let us assume that order $\varepsilon$ in Eq. (10) accepts the following solution:

$$
\begin{aligned}
w_{(m+1) 1}\left(x, T_{0}, T_{1}, T_{2}\right)= \\
{\left[A\left(T_{1}, T_{2}\right) \cdot e^{i \cdot \omega \cdot T_{0}}+c c\right] \cdot Y_{m+1}(x) . }
\end{aligned}
$$

In Eq. (14), $c c$ is the complex conjugate of the preceding terms, $\omega$ is the natural frequency, and $Y_{m+1}$ is the function describing the mode shape. By inserting Eq. (14) into Eqs. (10) and (13) and by assuming that $j=1$, one obtains the following differential equations and corresponding conditions:

$$
\begin{gathered}
Y_{m+1}^{\imath v}-\omega^{2} \cdot Y_{m+1}=\left\{\sum_{r=0}^{n} \int_{\eta_{r}}^{\eta_{r+1}} Y_{0}^{\prime} \cdot Y^{\prime}{ }_{r+1} \cdot d x\right\} \cdot Y^{\prime \prime}{ }_{0} \\
\left.Y_{1}\right|_{x=\eta_{0}}=\left.Y^{\prime \prime}{ }_{1}\right|_{x=\eta_{0}}=\left.Y_{n+1}\right|_{x=\eta_{n+1}}=\left.Y^{\prime \prime}{ }_{n+1}\right|_{x=\eta_{n+1}}=0 \\
\left.Y_{p}\right|_{x=\eta_{p}}=\left.Y_{p+1}\right|_{x=\eta_{p}}, \\
\left.Y_{p}^{\prime}\right|_{x=\eta_{p}}=\left.Y^{\prime}{ }_{p+1}\right|_{x=\eta_{p}}, \\
\left.Y_{p}^{\prime \prime}\right|_{x=\eta_{p}}=\left.Y_{p+1}^{\prime \prime}\right|_{x=\eta_{p}}, \\
\left.\left(Y^{\prime \prime \prime}{ }_{p}-Y^{\prime \prime \prime}{ }_{p+1}-\tau_{p} \cdot Y_{p}\right)\right|_{x=\eta_{p}}=0
\end{gathered}
$$

To be able to find the solution at the order $\varepsilon_{2}$ of the perturbation series, equality of $D_{1} \cdot A\left(T_{1}, T_{2}\right)=0$ must be provided. This results in assumption of $A=A\left(T_{2}\right)$ and means that there is no dependence on $T_{1}$ at this order. By inserting Eq. (14) into Eq. (11), the following solution is suitable at this order:

$$
\begin{aligned}
& w_{(m+1) 2}(x, T 2)= \\
& {\left[A^{2} \cdot e^{2 \cdot i \cdot \omega \cdot T_{0}}+c c\right] \cdot \varphi_{(m+1) 1}(x)+2 \cdot A \cdot \bar{A} \cdot \varphi_{(m+1) 2}(x) .}
\end{aligned}
$$

Substituting Eq. (16) into both Eq. (11) and (13) while keeping in mind that $j=2$, yields the following equations and condi- 
tions:

$$
\begin{aligned}
& \varphi_{(m+1) 1}^{\imath v}-4 \cdot \omega^{2} \cdot \varphi_{(m+1) 1}= \\
& \left\{\sum_{r=0}^{n} \int_{\eta_{r}}^{\eta_{r+1}} Y_{0}^{\prime} \cdot \varphi_{(r+1) 1}^{\prime} d x\right\} \cdot Y^{\prime \prime}{ }_{0} \\
& +\frac{1}{2} \cdot\left\{\sum_{r=0}^{n} \int_{\eta_{r}}^{\eta_{r+1}} Y_{r+1}^{\prime 2} d x\right\} \cdot Y^{\prime \prime}{ }_{0} \\
& +\left\{\sum_{r=0}^{n} \int_{\eta_{r}}^{\eta_{r+1}} Y_{0}^{\prime} \cdot Y^{\prime}{ }_{r+1} d x\right\} \cdot Y^{\prime \prime}{ }_{m+1} \\
& \varphi_{(m+1) 2}^{\imath v}=\left\{\sum_{r=0}^{n} \int_{\eta_{r}}^{\eta_{r+1}} Y^{\prime}{ }_{0} \cdot \varphi^{\prime}{ }_{(r+1) 2} d x\right\} \cdot Y^{\prime \prime}{ }_{0} \\
& +\frac{1}{2} \cdot\left\{\sum_{r=0}^{n} \int_{\eta_{r}}^{\eta_{r+1}} Y_{r+1}^{\prime 2} d x\right\} \cdot Y^{\prime \prime}{ }_{0} \\
& +\left\{\sum_{r=0}^{n} \int_{\eta_{r}}^{\eta_{r+1}} Y_{0}^{\prime} \cdot Y_{r+1}^{\prime} d x\right\} \cdot Y^{\prime \prime}{ }_{m+1} ; \\
& \left.\varphi_{p h}\right|_{x=\eta_{p}}=\left.\varphi_{(p+1) h}\right|_{x=\eta_{p}}, \\
& \left.\varphi_{p h}^{\prime}\right|_{x=\eta_{p}}=\left.\varphi_{(p+1) h}^{\prime}\right|_{x=\eta_{p}}, \\
& \left.\varphi^{\prime \prime}{ }_{p h}\right|_{x=\eta_{p}}=\left.\varphi^{\prime \prime}{ }_{(p+1) h}\right|_{x=\eta_{p}}, \\
& \left.\left(\varphi_{p h}^{\prime \prime \prime}-\varphi^{\prime \prime \prime}{ }_{(p+1) h}-\tau_{p} \cdot \varphi_{p h}\right)\right|_{x=\eta_{p}}=0 \\
& \left.\varphi_{1 h}\right|_{x=\eta_{0}}=\left.\varphi^{\prime \prime}{ }_{1 h}\right|_{x=\eta_{0}} \\
& =\left.\varphi_{(n+1) h}\right|_{x=\eta_{n+1}}=\left.\varphi^{\prime \prime}{ }_{(n+1) h}\right|_{x=\eta_{n+1}}=0 \\
& h=1,2 .
\end{aligned}
$$

At the last order $\left(\varepsilon_{3}\right)$ of the perturbation series, having substituted Eqs. (14) to (16) into Eq. (12), the resulting equation will accept the solution of the following separated form as secular and nonsecular terms:

$$
\begin{aligned}
& w_{(m+1) 3}\left(x, T_{0}, T_{2}\right)= \\
& \quad \phi_{m+1}\left(x, T_{2}\right) \cdot e^{i \cdot \omega \cdot T_{0}}+W_{m+1}\left(x, T_{2}\right)+c c ;
\end{aligned}
$$

where $W_{m+1}\left(x, T_{2}\right)$ corresponds to the solution for the nonsecular terms, and cc corresponds to the complex conjugate of the preceding terms. Let us take the excitation frequency as below:

$$
\Omega=\omega+\varepsilon^{2} \cdot \sigma
$$

where $\sigma$ is the detuning parameter denoting closeness of the forcing frequency to the natural frequency. By inserting Eqs. (20) and (21) into Eqs. (12) and (13), taking in mind $j=3$, and eliminating the secular terms, one obtains the differential equations and conditions in Eqs. (22) and (23) (see top of the next page).
In order to have a solution for Eqs. (22) and (23), a solvability condition must be satisfied for this nonhomogenous equation (see details in Refs. Nayfeh ${ }^{37,38}$ ). Applying the solvability condition for Eqs. (22) and (23), one obtains following equations:

$$
2 \cdot i \cdot \omega \cdot(\dot{A}+\mu \cdot A)+A^{2} \cdot \bar{A} \cdot \Gamma=\frac{1}{2} \cdot f \cdot e^{i \cdot \sigma \cdot T_{2}} .
$$

In Appendix (A.2), one can see normalization and simplification done for $\mathrm{Eq}$ (24).

By substituting the polar forms, we get:

$$
A\left(T_{2}\right)=\frac{1}{2} \cdot a \cdot e^{i \cdot \theta}, \quad \bar{A}\left(T_{2}\right)=\frac{1}{2} \cdot a \cdot e^{-i \cdot \theta}, \quad \theta=\theta\left(T_{2}\right)
$$

into Eq. (24), and separating real and imaginary parts, one obtains following equations:

$\mu \cdot a+\dot{a}=\frac{1}{2 \cdot . \omega} \cdot f \cdot \sin \gamma, \quad-a \cdot \dot{\theta}+\lambda \cdot a^{3}=\frac{1}{2 \cdot \omega} \cdot f \cdot \cos \gamma$

These equations can be defined as amplitude-phase modulation equations and consist of the real amplitude $a$ and phase $\theta$. The simplifications that were done can be seen in Appendix (A.3). Here, we have defined $\lambda$ as nonlinearity effect of the system.

In undamped free vibrations, the terms $f, \mu$, and $\sigma$ were taken as zero. $a=a_{0}$ is assumed because $\dot{a}=0$ is taken for the steady-state solutions. This indicates that the amplitude of vibration is constant. Therefore, the nonlinear frequency was defined as:

$$
\omega n l=\omega+\dot{\theta}=\omega+\lambda \cdot a_{0}^{2} .
$$

In damped-forced vibrations for the steady-state region, $\dot{a}$ and $\dot{\gamma}$ can be taken as zero and denote no change in amplitude and phase with time. Thus, eliminating $\gamma$ from Eq. (26), one can obtain the detuning parameter $(\sigma)$ as below:

$$
\sigma=\lambda \cdot a_{0}^{2} \pm \sqrt{\left(\frac{f}{2 \cdot a_{0} \cdot \omega}\right)^{2}-\mu^{2}} .
$$

\subsection{Three to One Internal Resonance}

In this section, the 3:1 internal resonance case between the kth and sth mode of the system will be discussed. For the solution, a two-mode expansion is considered because of the interaction between the two modes. In Eq. (9), the first order of the perturbation series is assumed to have a solution as below:

$$
\begin{aligned}
& w_{(m+1) 1}\left(x, T_{0}, T_{1}, T_{2}\right)= \\
& \quad\left[A_{k}\left(T_{1}, T_{2}\right) \cdot e^{i \cdot \omega_{k} \cdot T_{0}}+c c\right] \cdot Y_{(m+1) k}(x) \\
& \quad+\left[A_{s}\left(T_{1}, T_{2}\right) \cdot e^{i \cdot \omega_{s} \cdot T_{0}}+c c\right] \cdot Y_{(m+1) s}(x) .
\end{aligned}
$$

Inserting this solution into Eqs. (10) and (13), one obtains the following equations and conditions belonging to the $k$ th and $s$ th modes as simplified in letter $g$ :

$$
\begin{aligned}
Y_{(m+1) g}^{\imath v}- & \omega_{g}^{2} \cdot Y_{(m+1) g}= \\
& \left\{\sum_{r=0}^{n} \int_{\eta_{r}}^{\eta_{r+1}} Y_{0}^{\prime} \cdot Y^{\prime}{ }_{(r+1) g} \cdot d x\right\} \cdot Y^{\prime \prime}{ }_{0}
\end{aligned}
$$




$$
\begin{aligned}
& \phi_{m+1}^{\imath v}-\omega^{2} \cdot \phi_{m+1}-\left\{\sum_{r=0}^{n} \int_{\eta_{r}}^{\eta_{r+1}} Y_{0}^{\prime} \cdot \phi_{r+1}^{\prime} d x\right\} \cdot Y^{\prime \prime}{ }_{0}=-2 \cdot i \cdot \omega \cdot(\dot{A}+\mu \cdot A) \cdot Y_{m+1}+\frac{1}{2} \cdot F_{m+1} \cdot e^{i . \sigma \cdot T_{2}} \\
& +A^{2} \bar{A} \cdot\left[\sum_{r=0}^{n} \int_{\eta_{r}}^{\eta_{r+1}} Y_{0}^{\prime} \cdot Y^{\prime}{ }_{r+1} d x \cdot\left[\varphi^{\prime \prime}{ }_{(m+1) 1}+2 \cdot \varphi^{\prime \prime}{ }_{(m+1) 2}\right]\right. \\
& +\left\{\sum_{r=0}^{n} \int_{\eta_{r}}^{\eta_{r+1}} Y_{r+1}^{\prime} \cdot \varphi_{(r+1) 1}^{\prime} d x+2 \cdot \sum_{r=0}^{n} \int_{\eta_{r}}^{\eta_{r+1}} Y_{r+1}^{\prime} \cdot \varphi_{(r+1) 2}^{\prime} d x\right\} \cdot Y^{\prime \prime}{ }_{0} \\
& \left.+\left\{\frac{3}{2} \cdot \sum_{r=0}^{n} \int_{\eta_{r}}^{\eta_{r+1}} Y_{r+1}^{\prime 2} d x+\sum_{r=0}^{n} \int_{\eta_{r}}^{\eta_{r+1}} Y^{\prime}{ }_{0} \cdot \varphi^{\prime}{ }_{(r+1) 1} d x+2 \cdot \sum_{r=0}^{n} \int_{\eta_{r}}^{\eta_{r+1}} Y^{\prime}{ }_{0} \cdot \varphi^{\prime}{ }_{(r+1) 2} d x\right\} \cdot Y^{\prime \prime}{ }_{m+1}\right] \\
& \left.\phi_{p}\right|_{x=\eta_{p}}=\left.\phi_{p+1}\right|_{x=\eta_{p}},\left.\quad \phi_{p}^{\prime}\right|_{x=\eta_{p}}=\left.\phi_{p+1}^{\prime}\right|_{x=\eta_{p}},\left.\quad \phi_{p}^{\prime \prime}\right|_{x=\eta_{p}}=\left.\phi_{p+1}^{\prime \prime}\right|_{x=\eta_{p}},\left.\quad\left(\phi^{\prime \prime \prime}{ }_{p}-\phi^{\prime \prime \prime}{ }_{p+1}-\tau_{p} \cdot \phi_{p}\right)\right|_{x=\eta_{p}}=0, \\
& \left.\phi_{1}\right|_{x=\eta_{0}}=\left.\phi_{1}{ }^{\prime \prime}\right|_{x=\eta_{0}}=\left.\phi_{n+1}\right|_{x=\eta_{n+1}}=\left.\phi_{n+1}{ }^{\prime \prime}\right|_{x=\eta_{n+1}}=0 \text {. }
\end{aligned}
$$

$$
\begin{aligned}
\left.Y_{p g}\right|_{x=\eta_{p}} & =\left.Y_{(p+1) g}\right|_{x=\eta_{p}}, \\
\left.Y_{p g}^{\prime}\right|_{x=\eta_{p}} & =\left.Y^{\prime}{ }_{(p+1) g}\right|_{x=\eta_{p}}, \\
\left.Y^{\prime \prime}{ }_{p g}\right|_{x=\eta_{p}} & =\left.Y^{\prime \prime}{ }_{(p+1) g}\right|_{x=\eta_{p}}, \\
\left(Y_{p g}^{\prime \prime \prime}-Y_{(p+1) g}^{\prime \prime \prime}\right. & \left.-\tau_{p} \cdot Y_{p g}\right)\left.\right|_{x=\eta_{p}}=0, \\
\left.Y_{1 g}\right|_{x=\eta_{0}}=\left.Y^{\prime \prime}{ }_{1 g}\right|_{x=\eta_{0}} & =\left.Y_{(n+1) g}\right|_{x=\eta_{n+1}} \\
& =\left.Y_{(n+1) g}^{\prime \prime}\right|_{x=\eta_{n+1}}=0, \\
g & =k, s .
\end{aligned}
$$

In order to obtain the solutions at the order $\varepsilon_{2}$ of perturbation series, it should be assumed that $A_{k}=A_{k}\left(T_{2}\right), A_{s}=$ $A_{m}\left(T_{2}\right)$. This necessity requires no dependence on $T_{1}$ in this order. Inserting Eqs. (30) and (31) into Eq. (11), the equation at order $\varepsilon_{2}$ has the following solution:

$$
\begin{aligned}
& w_{(m+1) 2}\left(x, T_{2}\right)= \\
& {\left[A_{k}^{2} \cdot e^{2 \cdot i \cdot \omega_{k} \cdot T_{0}}+c c\right] \cdot \varphi_{(m+1) 1}(x)} \\
& +\left[A_{s}^{2} \cdot e^{2 \cdot i \cdot \omega_{s} \cdot T_{0}}+c c\right] \cdot \varphi_{(m+1) 2}(x) \\
& +\left[A_{k} \cdot A_{s} \cdot e^{i \cdot\left(\omega_{k}+\omega_{s}\right) \cdot T_{0}}+c c\right] \cdot \varphi_{(m+1) 3}(x) \\
& +\left[A_{s} \cdot \overline{A_{k}} \cdot e^{i \cdot\left(\omega_{s}-\omega_{k}\right) \cdot T_{0}}+c c\right] \cdot \varphi_{(m+1) 4}(x) \\
& +2 \cdot A_{s} \cdot \overline{A_{s}} \cdot \varphi_{(m+1) 5}(x) \\
& +2 \cdot A_{k} \cdot \overline{A_{k}} \cdot \varphi_{(m+1) 6}(x) .
\end{aligned}
$$

Inserting Eq. (32) into Eqs. (11) and (13), and separating the $k$ th and $s$ th modes, one can obtain the following differential equations:

$$
\begin{aligned}
& \varphi_{(m+1) 1}^{\imath v}-\kappa_{1}{ }^{4} \cdot \varphi_{(m+1) 1} \\
& -\left\{\sum_{r=0}^{s} \int_{\eta_{r}}^{\eta_{r+1}} Y_{0}^{\prime} \cdot \varphi_{(r+1) 1}^{\prime} d x\right\} \cdot Y^{\prime \prime}{ }_{0} \\
& =\frac{1}{2} \cdot\left\{\sum_{r=0}^{s} \int_{\eta_{r}}^{\eta_{r+1}} Y_{(r+1) k}^{\prime 2} d x\right\}, \\
& \cdot Y_{0}{ }^{\prime \prime}\left\{\sum_{r=0}^{s} \int_{\eta_{r}}^{\eta_{r+1}} Y_{0}{ }^{\prime} \cdot Y_{(r+1) k}{ }^{\prime} d x\right\} \cdot Y_{(m+1) k}{ }^{\prime \prime} \text {; }
\end{aligned}
$$

$$
\begin{aligned}
\varphi_{(m+1) 2}^{\imath v}- & \kappa_{2}{ }^{4} \cdot \varphi_{(m+1) 2} \\
- & \left\{\sum_{r=0}^{s} \int_{\eta_{r}}^{\eta_{r+1}} Y_{0}^{\prime} \cdot \varphi^{\prime}{ }_{(r+1) 2} d x\right\} \cdot Y^{\prime \prime}{ }_{0} \\
& =\frac{1}{2} \cdot\left\{\sum_{r=0}^{s} \int_{\eta_{r}}^{\eta_{r+1}} Y_{(r+1) s}^{\prime 2} d x\right\} \cdot Y^{\prime \prime}{ }_{0} \\
& +\left\{\sum_{r=0}^{s} \int_{\eta_{r}}^{\eta_{r+1}} Y^{\prime}{ }_{0} \cdot Y^{\prime}{ }_{(r+1) s} d x\right\} \cdot Y^{\prime \prime}{ }_{(m+1) s},
\end{aligned}
$$$$
\varphi_{(m+1) 3}^{\imath v}-\kappa_{3}{ }^{4} \cdot \varphi_{(m+1) 3}
$$$$
-\left\{\sum_{r=0}^{s} \int_{\eta_{r}}^{\eta_{r+1}} Y_{0}^{\prime} \cdot \varphi_{(r+1) 3}^{\prime} d x\right\} \cdot Y^{\prime \prime}{ }_{0}
$$$$
=\left\{\sum_{r=0}^{s} \int_{\eta_{r}}^{\eta_{r+1}} Y^{\prime}{ }_{(r+1) k} \cdot Y^{\prime}{ }_{(r+1) s} d x\right\} \cdot Y^{\prime \prime}{ }_{0}
$$$$
+\left\{\sum_{r=0}^{s} \int_{\eta_{r}}^{\eta_{r+1}} Y_{0}^{\prime} \cdot Y^{\prime}{ }_{(r+1) k} d x\right\} \cdot Y^{\prime \prime}{ }_{(m+1) s}
$$ 


$$
\begin{aligned}
& +\left\{\sum_{r=0}^{s} \int_{\eta_{r}}^{\eta_{r+1}} Y_{0}^{\prime} \cdot Y_{(r+1) s}^{\prime} d x\right\} \cdot Y_{(m+1) k}^{\prime \prime}, \\
& \varphi_{(m+1) 4}^{\imath v}-\kappa_{4}{ }^{4} \cdot \varphi_{(m+1) 4} \\
& -\left\{\sum_{r=0}^{s} \int_{\eta_{r}}^{\eta_{r+1}} Y_{0}^{\prime} \cdot \varphi_{(r+1) 4}^{\prime} d x\right\} \cdot Y^{\prime \prime}{ }_{0} \\
& =\left\{\sum_{r=0}^{s} \int_{\eta_{r}}^{\eta_{r+1}} Y_{(r+1) k}^{\prime} \cdot Y^{\prime}{ }_{(r+1) s} \cdot d x\right\} \cdot Y^{\prime \prime}{ }_{0} \\
& +\left\{\sum_{r=0}^{s} \int_{\eta_{r}}^{\eta_{r+1}} Y_{0}^{\prime} \cdot Y_{(r+1) k}^{\prime} d x\right\} \cdot Y^{\prime \prime}{ }_{(m+1) s} \\
& +\left\{\sum_{r=0}^{s} \int_{\eta_{r}}^{\eta_{r+1}} Y_{0}^{\prime} \cdot Y_{(r+1) s}^{\prime} d x\right\} \cdot Y^{\prime \prime}{ }_{(m+1) k}, \\
& \varphi_{(m+1) 5}^{\imath v}-\left\{\sum_{r=0}^{s} \int_{\eta_{r}}^{\eta_{r+1}} Y_{0}^{\prime} \cdot \varphi^{\prime}{ }_{(r+1) 5} d x\right\} \cdot Y_{0}^{\prime \prime} \\
& =\frac{1}{2} \cdot\left\{\sum_{r=0}^{s} \int_{\eta_{r}}^{\eta_{r+1}} Y_{(r+1) k}^{\prime 2} d x\right\} \cdot Y_{0}^{\prime \prime} \\
& +\left\{\sum_{r=0}^{s} \int_{\eta_{r}}^{\eta_{r+1}} Y_{0}^{\prime} \cdot Y^{\prime}{ }_{(r+1) k} d x\right\} \cdot Y^{\prime \prime}{ }_{(m+1) k}, \\
& \varphi_{(m+1) 6}^{\imath v}-\left\{\sum_{r=0}^{s} \int_{\eta_{r}}^{\eta_{r+1}} Y_{0}^{\prime} \cdot \varphi_{(r+1) 6}^{\prime} d x\right\} \cdot Y^{\prime \prime}{ }_{0} \\
& =\frac{1}{2} \cdot\left\{\sum_{r=0}^{s} \int_{\eta_{r}}^{\eta_{r+1}} Y_{(r+1) s}^{\prime 2} d x\right\} \cdot Y^{\prime \prime}{ }_{0} \\
& +\left\{\sum_{r=0}^{s} \int_{\eta_{r}}^{\eta_{r+1}} Y_{0}^{\prime} \cdot Y^{\prime}{ }_{(r+1) s} \cdot d x\right\} \cdot Y^{\prime \prime}{ }_{(m+1) s} .
\end{aligned}
$$

The following conditions were also obtained:

$$
\begin{gathered}
\left.\varphi_{p v}\right|_{x=\eta_{p}}=\left.\varphi_{(p+1) v}\right|_{x=\eta_{p}}, \\
\left.\varphi_{p v}^{\prime}\right|_{x=\eta_{p}}=\left.\varphi_{(p+1) v}^{\prime}\right|_{x=\eta_{p}} \\
\left.\varphi_{p v}^{\prime \prime}\right|_{x=\eta_{p}}=\left.\varphi^{\prime \prime}{ }_{(p+1) v}\right|_{x=\eta_{p}}, \\
\left.\left(\varphi_{p 1}^{\prime \prime \prime}-\varphi_{(p+1) 1}^{\prime \prime \prime}-\tau_{p} \cdot \varphi_{p 1}\right)\right|_{x=\eta_{p}}=0 \\
\left.\varphi_{1 v}\right|_{x=\eta_{0}}=\left.\varphi_{(s+1) v}\right|_{x=\eta_{s+1}}=0 \\
=\left.\varphi^{\prime \prime}{ }_{1 v}\right|_{x=\eta_{0}}=\left.\varphi_{(s+1) v}\right|_{x=\eta_{s+1}}=\varphi^{\prime . .6}
\end{gathered}
$$

The solutions at order $\varepsilon_{3}$ were similar to those of the first order and can be written as:

$$
\begin{aligned}
w_{(m+1) 3}\left(x, T_{0}, T_{2}\right) & \\
& =\phi_{(m+1) k} \\
& \left(x, T_{2}\right) \cdot e^{i \cdot \omega_{k} \cdot T_{0}} \\
& +\phi_{(m+1) s}\left(x, T_{2}\right) \cdot e^{i \cdot \omega_{s} \cdot T_{0}} \\
& +W_{m+1}\left(x, T_{0}, T_{2}\right)+c c .
\end{aligned}
$$

Thus, let us assume that the forcing frequency is close to the natural frequency of the kth mode, and there is an approximate ratio as three-to-one between the kth and sth modes as:

$$
\Omega=\omega_{k}+\varepsilon^{2} \cdot \sigma, \quad \omega_{s}=3 \cdot \omega_{k}+\varepsilon^{2} \cdot q
$$

In this case, by substituting Eqs. (35) and (36) into Eqs. (12) and (13) and eliminating non-secular terms, one obtains the following equations and conditions:

$$
\begin{aligned}
& \phi_{(m+1) k}^{\imath v}-\omega_{k}^{2} \cdot \phi_{(m+1) k} \\
& -\left\{\sum_{r=0}^{n} \int_{\eta_{r}}^{\eta_{r+1}} Y^{\prime}{ }_{0} \cdot \phi^{\prime}{ }_{(r+1) k} d x\right\} \cdot Y^{\prime \prime}{ }_{0} \\
& =-2 \cdot i \cdot \omega_{k} \cdot\left(\dot{A}_{k}+\mu \cdot A_{k}\right) \cdot Y_{(m+1) k}+\frac{1}{2} \cdot e^{i \cdot \sigma \cdot T_{2}} \cdot F_{m+1} \\
& +A_{k}^{2} \cdot \overline{A_{k}} \cdot \Gamma_{(m+1) 1}{ }^{(k)}+A_{s} \cdot \overline{A_{s}} \cdot A_{k} \cdot \Gamma_{(m+1) 2}{ }^{(k)} \\
& +{\overline{A_{k}}}^{2} \cdot A_{s} \cdot e^{i \cdot q \cdot T_{2}} \cdot \Gamma_{(m+1) 3}{ }^{(k)} ; \\
& \phi_{(m+1) s}^{\imath v}-\omega_{s}^{2} \cdot \phi_{(m+1) s} \\
& -\left\{\sum_{r=0}^{n} \int_{\eta_{r}}^{\eta_{r+1}} Y_{0}^{\prime} \cdot \phi_{(r+1) s}^{\prime} d x\right\} \cdot Y^{\prime \prime}{ }_{0} \\
& =-2 \cdot i \cdot \omega_{s} \cdot\left(\dot{A}_{s}+\mu \cdot A_{s}\right) \cdot Y_{(m+1) s} \\
& +A_{s}{ }^{2} \cdot \overline{A_{s}} \cdot \Gamma_{(m+1) 1}{ }^{(s)}+A_{k} \cdot \overline{A_{k}} \cdot A_{s} \cdot \Gamma_{(m+1) 2}{ }^{(s)} \\
& +A_{k}^{3} \cdot e^{-i \cdot q \cdot T_{2}} \cdot \Gamma_{(m+1) 3}^{(s)} \text {; } \\
& \left.\phi_{p g}\right|_{x=\eta_{p}}=\left.\phi_{(p+1) g}\right|_{x=\eta_{p}}, \\
& \left.\phi_{p g}^{\prime}\right|_{x=\eta_{p}}=\left.\phi_{(p+1) g}^{\prime}\right|_{x=\eta_{p}}, \\
& \left.\phi_{p g}^{\prime \prime}\right|_{x=\eta_{p}}=\left.\phi^{\prime \prime}{ }_{(p+1) g}\right|_{x=\eta_{p}}, \\
& \left.\left(\phi_{p g}^{\prime \prime \prime}-\phi_{(p+1) g}^{\prime \prime \prime}-\tau_{p} \cdot \phi_{p g}\right)\right|_{x=\eta_{p}}=0, \\
& \left.\phi_{1 g}\right|_{x=\eta_{0}}=\left.\phi^{\prime \prime}{ }_{1 g}\right|_{x=\eta_{0}} \\
& =\left.\phi_{(n+1) g}\right|_{x=\eta_{n+1}}=\left.\phi^{\prime \prime}{ }_{(n+1) g}\right|_{x=\eta_{n+1}}=0 ; \\
& g=k, s \text {. }
\end{aligned}
$$

$\Gamma$ 's in the above equations are defined in Appendix (??).

If the solvability condition (see Nayfeh for further details ${ }^{37,38}$ ) is applied in order to solve Eqs. (37), (38) and (39) the following equations were obtained: 


$$
\begin{array}{r}
2 \cdot i \cdot \omega_{k} \cdot\left(\dot{A}_{k}+\mu \cdot A_{k}\right)-\lambda_{1}{ }^{(k)} \cdot A_{k}{ }^{2} \cdot \overline{A_{k}}-\lambda_{2}{ }^{(k)} \cdot A_{s} \cdot \overline{A_{s}} \cdot A_{k} \\
-\lambda_{3}{ }^{(k)} \cdot{\overline{A_{k}}}^{2} \cdot A_{s} \cdot e^{i \cdot q \cdot T_{2}}=\frac{1}{2} \cdot f \cdot e^{i \cdot \sigma \cdot T_{2}} \\
2 \cdot i \cdot \omega_{s} \cdot\left(\dot{A}_{s}+\mu \cdot A_{s}\right)-\lambda_{1}{ }^{(s)} \cdot A_{s}{ }^{2} \cdot \overline{A_{s}}-\lambda_{2}{ }^{(s)} \cdot A_{k} \cdot \overline{A_{k}} \cdot A_{s} \\
-\lambda_{3}{ }^{(s)} \cdot A_{k}{ }^{3} \cdot e^{-i \cdot q \cdot T_{2}}=0
\end{array}
$$

In Appendix (A.5), one can see normalizations and simplifications done for Eq. (40). In order to rewrite the equations in real amplitude form, the following complex transformations were used:

$$
A_{k}=\frac{1}{2} \cdot a_{k} \cdot e^{i \cdot \theta_{k}}, \quad A_{s}=\frac{1}{2} \cdot a_{s} \cdot e^{i \cdot \theta_{s}} .
$$

Using Eq. (41), the following equations were obtained:

$$
\begin{gathered}
i \cdot \omega_{k} \cdot\left(\dot{a}_{k}+\mu \cdot a_{k}\right)-\omega_{k} \cdot a_{k} \cdot \dot{\theta}_{k}-\frac{\lambda_{1}{ }^{(k)}}{8} \cdot a_{k}{ }^{3} \\
-\frac{\lambda_{2}{ }^{(k)}}{8} \cdot a_{s}{ }^{2} \cdot a_{k}-\frac{\lambda_{3}{ }^{(k)}}{8} \cdot a_{k}{ }^{2} \cdot a_{s} \cdot e^{i \cdot \beta}=\frac{1}{2} \cdot f \cdot e^{i \cdot \gamma} \\
i \cdot \omega_{s} \cdot\left(\dot{a}_{s}+\mu \cdot a_{s}\right)-\omega_{s} \cdot a_{s} \cdot \dot{\theta}_{s}-\frac{\lambda_{1}{ }^{(s)}}{8} \cdot a_{s}{ }^{3} \\
\quad-\frac{\lambda_{2}{ }^{(s)}}{8} \cdot a_{k}{ }^{2} \cdot a_{s}-\frac{\lambda_{3}{ }^{(s)}}{8} \cdot a_{k}{ }^{3} \cdot e^{-i \cdot \beta}=0
\end{gathered}
$$

Simplifications for $\gamma$ and $\beta$ done here can be seen in Appendix (A.6). By separating the real and imaginary parts of Eq. (42), one obtains the following four equations:

$$
\begin{gathered}
\omega_{k} \cdot\left(\dot{a}_{k}+\mu \cdot a_{k}\right)-\frac{\lambda_{3}{ }^{(k)}}{8} \cdot a_{k}{ }^{2} \cdot a_{s} \cdot \sin \beta=\frac{1}{2} \cdot f \cdot \sin \gamma \\
\omega_{s} \cdot\left(\dot{a}_{s}+\mu \cdot a_{s}\right)+\frac{\lambda_{3}{ }^{(s)}}{8} \cdot a_{k}{ }^{3} \cdot \sin \beta=0 \\
-\omega_{k} \cdot a_{k} \cdot(\sigma-\dot{\gamma})-\frac{\lambda_{1}{ }^{(k)}}{8} \cdot a_{k}{ }^{3}-\frac{\lambda_{2}{ }^{(k)}}{8} \cdot a_{s}{ }^{2} \cdot a_{k} \\
-\frac{\lambda_{3}{ }^{(k)}}{8} \cdot a_{k}{ }^{2} \cdot a_{s} \cdot \cos \beta=\frac{1}{2} \cdot f \cdot \cos \gamma \\
-\omega_{s} \cdot a_{s} \cdot(\dot{\beta}+3 \cdot(\sigma-\dot{\gamma})-q)-\frac{\lambda_{1}{ }^{(s)}}{8} \cdot a_{s}{ }^{3} \\
-\frac{\lambda_{2}{ }^{(s)}}{8} \cdot a_{k}{ }^{2} \cdot a_{s}-\frac{\lambda_{3}{ }^{(s)}}{8} \cdot a_{k}{ }^{3} \cdot \cos \beta=0
\end{gathered}
$$

Let us put the derivatives with respect to time to one side for determining the dynamic behavior of the system:

$$
\begin{gathered}
G_{1} \Rightarrow \dot{a}_{k}=\left\{\frac{1}{2} \cdot f \cdot \sin \gamma+\frac{\lambda_{3}{ }^{(k)}}{8} \cdot a_{k}{ }^{2} \cdot a_{s} \cdot \sin \beta\right. \\
\left.-\omega_{k} \cdot \mu \cdot a_{k}\right\} \cdot \frac{1}{\omega_{k}}, \\
G_{2} \Rightarrow \dot{a}_{s}=\left\{-\frac{\lambda_{3}{ }^{(s)}}{8} \cdot a_{k}{ }^{3} \cdot \sin \beta-\omega_{s} \cdot \mu \cdot a_{s}\right\} \cdot \frac{1}{\omega_{s}},
\end{gathered}
$$

$$
\begin{aligned}
& G_{3} \Rightarrow \dot{\gamma}=\left\{\frac{1}{2} \cdot f \cdot \cos \gamma+\frac{\lambda_{1}{ }^{(k)}}{8} \cdot a_{k}{ }^{3}+\frac{\lambda_{2}{ }^{(k)}}{8} \cdot a_{s}{ }^{2} \cdot a_{k}\right. \\
&+\left.\frac{\lambda_{3}{ }^{(k)}}{8} \cdot a_{k}{ }^{2} \cdot a_{s} \cdot \cos \beta+\omega_{k} \cdot a_{k} \cdot \sigma\right\} \cdot \frac{1}{\omega_{k} \cdot a_{k}}, \\
& G_{4} \Rightarrow \dot{\beta}=\left\{-\frac{\lambda_{1}{ }^{(s)}}{8} \cdot a_{s}{ }^{3}-\frac{\lambda_{2}{ }^{(s)}}{8} \cdot a_{k}{ }^{2} \cdot a_{s}-\frac{\lambda_{3}{ }^{(s)}}{8} \cdot a_{k}{ }^{3} \cdot \cos \beta\right. \\
&\left.-\omega_{s} \cdot a_{s} \cdot(3 \cdot(\sigma-\dot{\gamma})-q)\right\} \cdot \frac{1}{\omega_{s} \cdot a_{s}} \cdot
\end{aligned}
$$

In the steady state case of the system, it is assumed that there is no dependence on time. Therefore, taking $\dot{a}_{k}=\dot{a}_{s}=\dot{\gamma}=$ $\dot{\beta}=0$ in Eq. (36), the steady-state solutions can be found. The Jacobian matrix is constructed to determine the stability of fixed points:

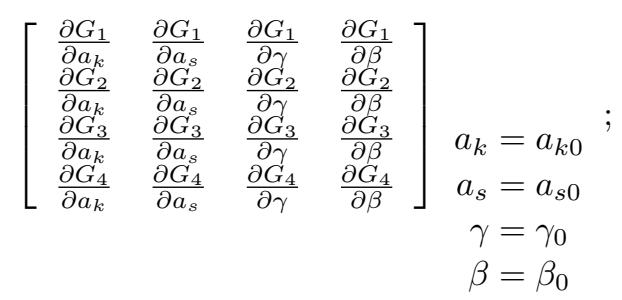

where terms with 0 indices define fixed points of the steady state. By evaluating the eigenvalues of the Jacobian matrix, stability is determined. If all eigenvalues of the Jacobian matrix have negative real parts, these fixed points were stable otherwise unstable.

\section{NUMERICAL RESULTS}

Let us assume the rise function of the curved beam has sinusoidal variation in the numeric analysis. Taking the dimensionless form of the curvature as $Y_{0}=\sin (\pi \cdot x)$, primary and three-to-one internal resonances were investigated in seperated sections as given below.

\subsection{Case of Primary Resonance}

Natural frequencies can be found by solving the linear problem and conditions in Eq. (15). The first five natural frequencies of the curved beam-spring system were given for two and three springs in Tabs. 1 and 2 respectively. The springs $\left(\tau_{p}\right)$, whose dimensionless sizes were selected as 10 and 100, were assumed to be placed at arbitrary points $\left(\eta_{p}\right)$ of the beam, making symmetric and asymmetric cases according to spring locations in these tables.

In order to find the approximate solutions to the mathematical model, the nonlinear problems were taken into consideration. For this purpose, Eq. (15), which corresponds to the linear part of the problem, is solved first. Then, nonlinearity coefficients $(\lambda)$ were obtained by using Eqs. (17) and (18). Nonlinearities $(\lambda)$ of the first mode were given in the cases of two and three springs in Tabs. 1 and 2 respectively. As seen on these tables, nonlinearities have positive and negative signs according to locations and magnitudes of the springs. If so, we 
Table 1. The first five natural frequencies and nonlinearity coefficients (/lambda) of the first mode for the beam resting on two springs.

\begin{tabular}{||c|c|c|c|c|c|c|c|c|c|}
\hline$\eta_{1}$ & $\eta_{2}$ & $t_{1}$ & $t_{2}$ & $\omega_{1}$ & $\omega_{2}$ & $\omega_{3}$ & $\omega_{4}$ & $\omega_{5}$ & $\lambda\left(\omega_{1}\right)$ \\
\hline & & 10 & 10 & 12.682 & 39.796 & 88.911 & 157.993 & 246.821 & -0.7409 \\
0.1 & 0.3 & 10 & 100 & 16.162 & 41.961 & 89.013 & 158.190 & 247.188 & 0.0819 \\
& & 100 & 10 & 13.266 & 40.560 & 89.570 & 158.208 & 247.187 & -0.5316 \\
\hline \multirow{3}{*}{0.1} & \multirow{2}{*}{0.5} & 10 & 10 & 12.960 & 39.566 & 89.013 & 157.971 & 246.821 & -0.6339 \\
& & 100 & 10 & 18.498 & 39.566 & 90.039 & 157.971 & 247.188 & 0.2872 \\
& & 10 & 10 & 12.563 & 40.326 & 89.668 & 158.467 & 247.188 & -0.4377 \\
0.1 & 0.7 & 10 & 100 & 16.204 & 49.795 & 88.911 & 157.993 & 246.821 & -0.7382 \\
& & 100 & 10 & 13.318 & 40.545 & 89.011 & 158.191 & 247.187 & 0.0868 \\
& & 10 & 10 & 13.379 & 39.709 & 88.950 & 158.509 & 247.187 & -0.5123 \\
0.3 & 0.5 & 10 & 100 & 18.796 & 39.709 & 89.797 & 157.936 & 246.821 & -0.4925 \\
& & 100 & 10 & 16.734 & 41.874 & 89.049 & 158.134 & 247.188 & 0.3045 \\
\hline \multirow{3}{*}{0.3} & \multirow{2}{*}{0.7} & 10 & 10 & 13.125 & 39.934 & 88.848 & 157.957 & 246.821 & -0.1503 \\
& & 10 & 100 & 16.614 & 42.051 & 88.948 & 158.156 & 247.187 & 0.1354 \\
& & 100 & 20.116 & 43.788 & 89.047 & 158.356 & 247.553 & 0.3629 \\
\hline
\end{tabular}

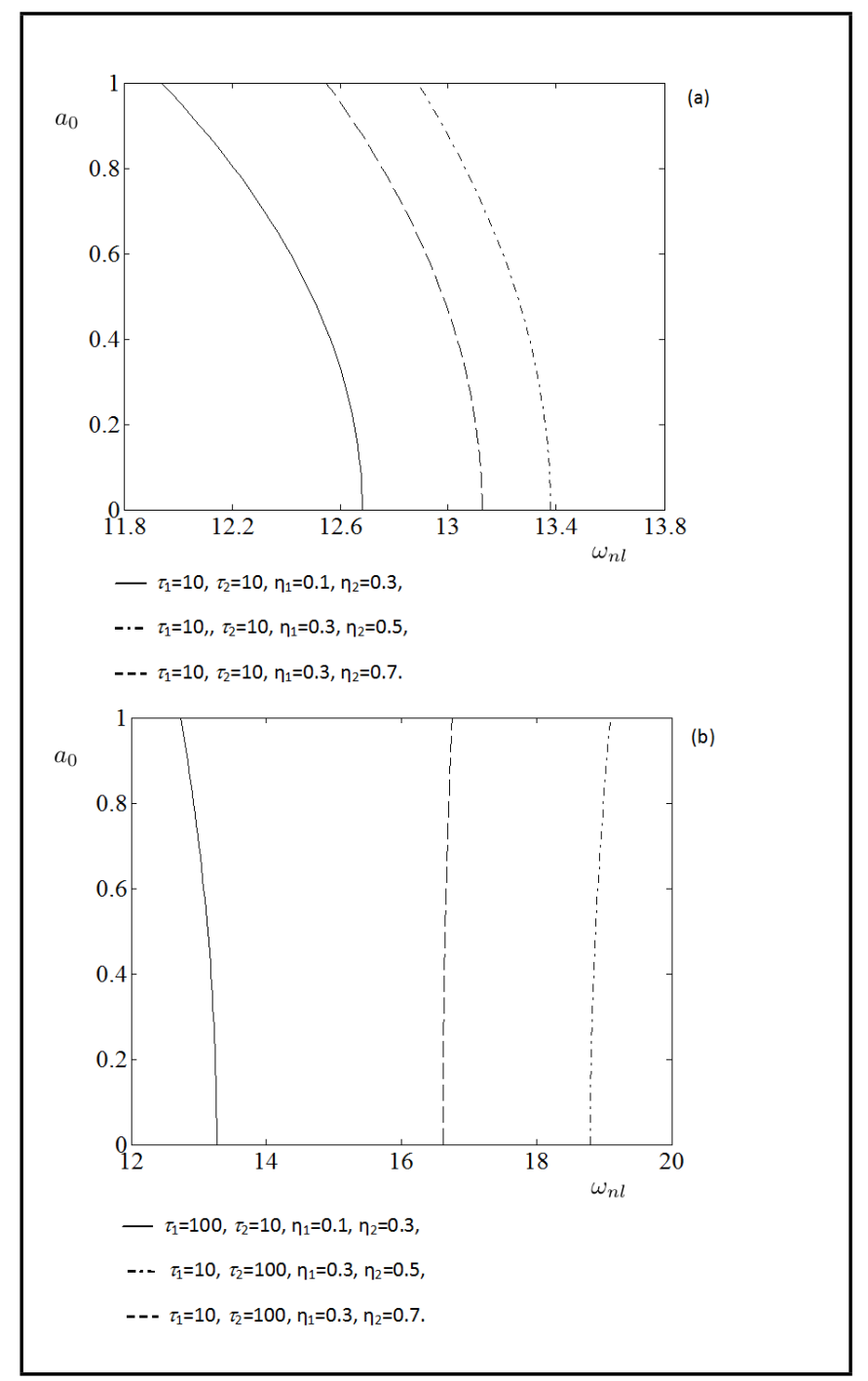

Figure 2. Nonlinear frequency-amplitude curves for beam resting on two springs.

were able to do hardening or softening behaviors of the system thanks to selecting suitable locations and magnitudes of the springs.

Undumped-free vibration behavior of the system is best seen in nonlinear frequency-amplitude curves. Nonlinear frequencies have a parabolic relationship with the maximum amplitude of the vibration as given in Eq. (27). These relations were drawn using curves for the first mode of vibrations in Figs. 2 to 3. The effects of magnitudes and locations of the springs to vibrations were determined through these curves.

In Fig. 2, the nonlinear frequency-amplitude curves were drawn for the beam resting on two springs. It was assumed that each spring had the same magnitude in Fig. 2a. By placing each of the springs in different locations, the effects of spring locations on nonlinear frequency-amplitude curves were drawn. In Fig. 2b, the springs were assumed to have different magnitudes. The effects of springs that had a higher stiffness than the others on nonlinear frequency were searched.

In Fig. 3, the nonlinear frequency-amplitude curves were drawn for the beam resting on three springs. Using spring stiffness at the same magnitude, different spring locations were used for each curve in Fig. 3a. Thus, the symmetric and asymmetric cases' effects on nonlinear frequency were also studied. Springs with different magnitudes were considered in Fig. 3b. The effects of springs on nonlinear frequencies were compared using high spring stiffness in magnitude. As seen from these figures, increases in number and in magnitude of the springs result in higher linear and nonlinear frequencies for the system. Selecting equal springs in magnitude in Fig. 4a and changes via locations of springs were searched in forcing frequencyresponse curves. Selecting different springs in magnitude, behaviors of the big one were searched in Fig. 4b.

Considering the case where there is damping and external excitation, nonlinear vibration behavior of the system could be understood via forcing frequency-response curves. When $f=1$ and $\mu=0.1$, some curves in Figs. 4 and 5 were drawn by means of Eq. (28). In these figures, only first modes of the tranverse vibrations were dealed. The case of two springs is taken into account in Fig. 4.

Considering the case of the three springs, curves in Fig. 5 were plotted. By placing the springs in different locations, symmetrical and asymmetrical cases were obtained. The effects of these cases on the curves were put forward. The maximum amplitudes of the vibrations decreased with increasing magnitudes and the number of springs. 


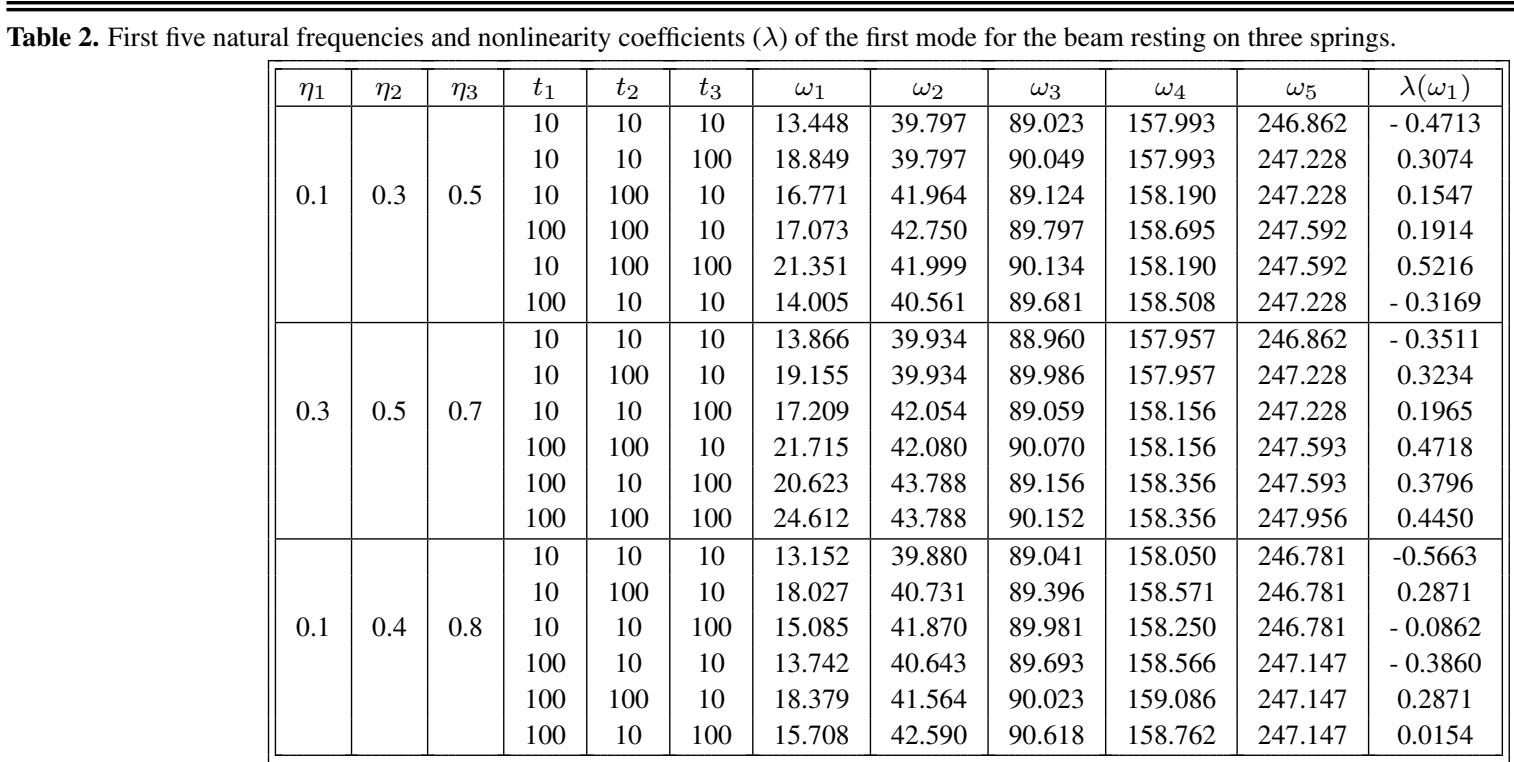

\subsection{Case of 3:1 internal resonances}

When browsing the table values for natural frequency of the system, one finds three-to-one ratio between natural frequencies of two different modes. Three-to-one internal resonance occurs between first and second modes of the curved beam for the case with two springs $\left(\tau_{1}=10, \tau_{2}=10, \eta_{1}=0.1, \eta_{2}=\right.$ $0.5)$ and three springs $\left(\tau_{1}=10, \tau_{2}=10, \tau_{3}=10, \eta_{1}=\right.$ $0.1, \eta_{2}=0.3, \eta_{3}=0.5$ ). The frequencies of the first and second modes were found to be $\omega_{1}=12.9603, \omega_{1}=39.5656$ for two springs, and $\omega_{1}=13.4475$ and $\omega_{2}=39.7965$ for three springs. Thus, resonance will happen and some energy of the first mode (externally forcing mode) will be trasferred to the second mode (internally forcing mode) during vibrations at these frequencies. Considering that the curved beam was resting on two springs or three springs, the forcing frequency-response curves were plotted for the externally forcing (first) mode in Fig. 6a and internally forcing (second) mode in Fig. 6b. Assuming $\mu=0.05$ and $f=1$ in Eq. (43), the frequencies and differences between them were considered, $\omega_{1}=12.9603, \omega_{2}=39.5656$, and $q=0.6847$ for the case of two springs and omega $a_{1}=13.4475, \omega_{2}=39.7965$, and $q=-0.5460$ for the case of three springs. For these values, one could evaluate fixed points by seperating stable and unstable solutions using Eqs. (44) and (45) and draw frequency-response curves. Seeing the curves in Fig. 6a, it can be concluded that that these systems have softening behavior. By means of detailed investigations in case of three springs, a more softening behavior and smaller maximum amplitude could be obtained when comparing case of two springs. Fig. 6b was plotted for internally forcing mode. As seen in detailed shot, maximum amplitude of the beam with two springs was pretty little according to case of three springs.

Considering curved beam resting on two springs or three springs, the forcing-response curves were plotted for the externally forcing mode in Fig. 7a and internally forcing mode in Fig. 7b. Taking into account the detuning parameter, $\sigma=$ -0.3788 for the case of two springs and $\sigma=-0.2625$ for the case of three springs, changes to $f$ were investigated in these figures. Other control parameters were the same in Fig. 6. As seen from the multi-variable region in Fig. 7, the case of two springs has more forcing $(f)$ gaps when comparing with case of three springs. These $f$ gaps is found as $[0.9990 /, 2.9785]$ for the case of two springs and [0.9995, /2.1105] for the case of three springs. Thus, internally forcing mode (second mode) was activated at $f \geq 0.9990$ for two springs and at $f \geq 0.9995$ for three springs.

\section{CONCLUSIONS}

In this study, nonlinear vibrations of a curved beam resting on multiple springs are investigated. The curved beam is based on the Euler-Bernoulli beam theory and is assumed to have an arbitrary rise function. Primary and 3:1 internal resonance cases are studied in nonlinear vibrations of the beam. Approximate analytical solutions to the problem are sought by using the method of multiple scales (MMS), which is a perturbation method. In these solution procedures, the linear problem of the system, which is first order, is solved. After that, the nonlinear problem is solved by adding the effects of nonlinearity, which comes from other orders, to the linear solution. Assuming a steady-state phase, a detailed analysis on free-undamped and forced-damped vibrations is carried out. Effects of magnitude, location, and the number of the springs on nonlinear vibrations is presented.

In case of primary resonance, nonlinearity effects of the curved beam-spring system has both positive and negative signs. Adjusting the number, location, and magnitudes of the springs attached under the beam enables us to make the system have softening behavior. Increasing the magnitude and number of the springs decreases the maximum amplitudes.

In case of three-to-one internal resonance, beams resting on two and three springs are considered. Three-to-one internal resonance between the first and second modes occur for both cases of spring replacements. From the carried investigations, energy transfer from the externally forcing mode (first mode) to the internally forcing mode (second mode) is much more in curved beams resting on three springs instead of two springs. 


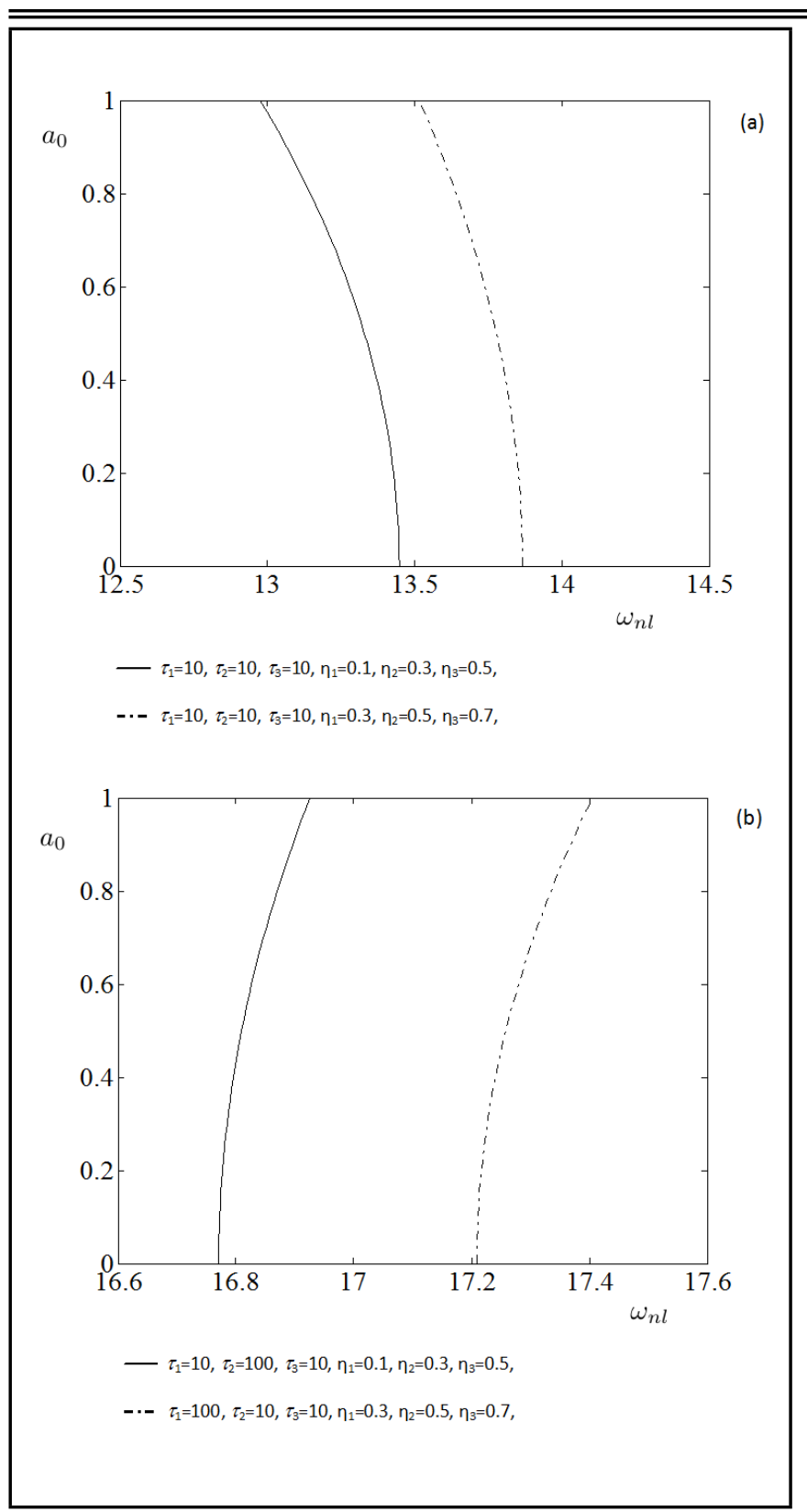

Figure 3. Nonlinear frequency-amplitude curves for beam resting on three springs.

\section{REFERENCES}

1 Nayfeh A. H., Mook D. T. Nonlinear oscillations, Willey, New York, (1979).

2 Cha P. D. Eigenvalues of a lineat elastica carrying lumped masses, springs and viscoues dampers, J. Sound Vib., 257 (4), 798-808, (2002).

3 Albarracin C. M., Zannier L., and Grossi R. O. Some observations in the dynamics of beams with intermediate supports, J. Sound Vib., 271, 475-480, (2004).

4 Wang J., Qiao P. Vibration of beams with arbitrary discontinuities and boundary conditions, J. Sound Vib., 308, 12-27, (2007).

5 Wiedemann S. M. Natural frequencies and mode shapes of arbitrary beam structures with arbitrary boundary conditions, J. Sound Vib., 300, 280-291, (2007).
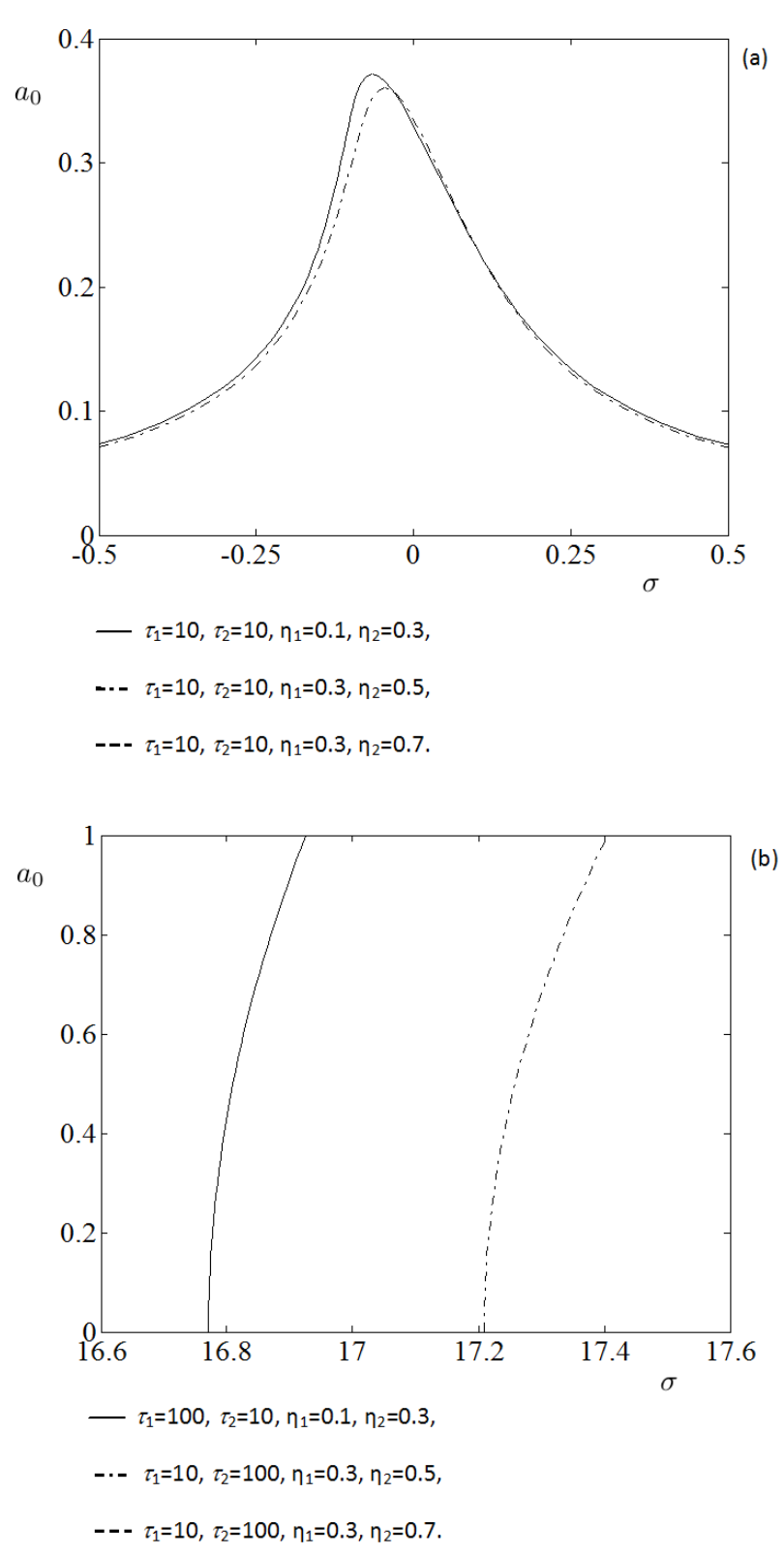

Figure 4. Forcing frequency-response curves for beam resting on two springs.

6 Huang D. T., Chen D. K. Dynamic characteristics of a structure with multiple attachments: A receptance approach, $J$. Sound Vib., 307, 941-952, (2007).

7 Kelly S. G., Srinivas S. Free vibrations of elastically connected stretched beams, J. Sound Vib., 326, 883-893, (2009).

8 Wang L., Ma J., Li L., and Peng J. Three-toone resonant responses of inextensional beams on the elastic foundation, J. Vib. Acoust., 135 (1), (2013). http://dx.doi.org/10.1115/1.40079

9 Rehfield L. W. Nonlinear flexural oscillation of shallow arches, AIAA Journal, 12, 91-93, (1974).

10 Singh P. N. and Ali S. M. J. Nonlinear vibration of a mod- 


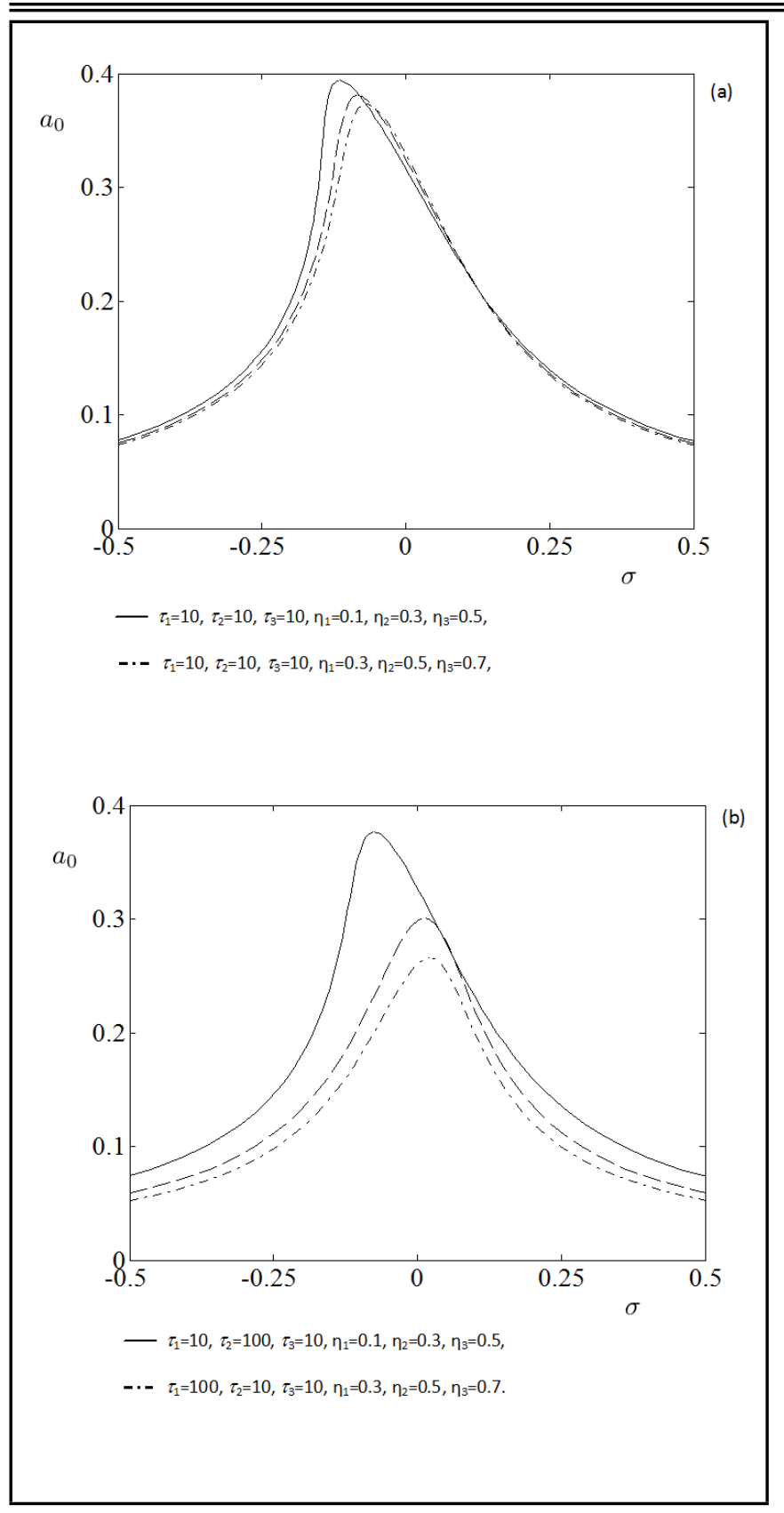

Figure 5. Forcing frequency-response curves for beam resting on three springs.

erately thick shallow arch, J. Sound Vib., 41, 275-282, (1975).

11 Hajianmaleki M., Qatu M. S. Vibrations of straight and curved composite beams, Comp. Struc, 100, 218-232, (2013).

12 Tien W. M., Sri Namachchivaya N. and Bajaj A. K. Nonlinear dynamics of a shallow arch under periodic excitationI. 1:2 internal resonance, Int. J. Non-Linear Mech., 29, 349366, (1994).

13 Krishnan A., Suresh Y. J. A simple cubic linear element for static and free vibration analyses of curved beam, Computers and Structures, 68, 473-489, (1998).

14 Oz H. R., Pakdemirli M., zkaya E., and Ylmaz M. Nonlinear vibrations of a slightly curved beam resting on a non- (a) externally forcing mode $\left(a_{1}\right)$

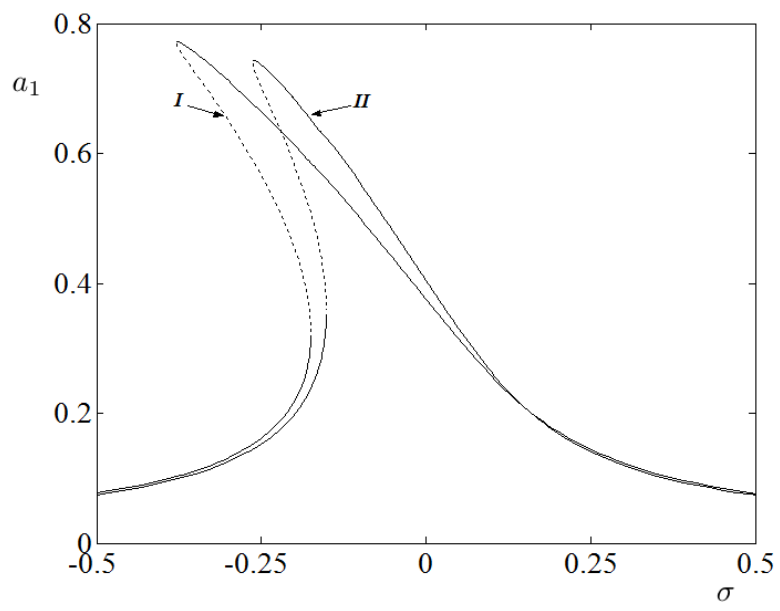

(b) internally forcing mode $\left(a_{2}\right)$

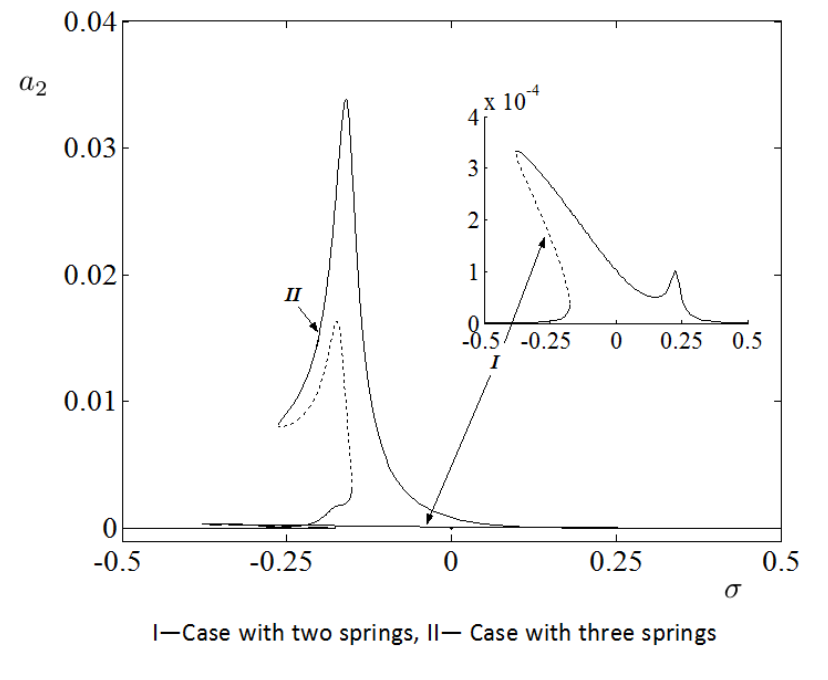

Figure 6. Forcing frequency-response curves.

linear elastic foundation, J. Sound Vib., 212 (2), 295-309, (1998).

15 Lin S. M. Exact solutions for extensible circular curved Timoshenko beams with nonhomogeneous elastic boundary conditions, Acta Mech., 130, 67-79, (1998).

16 Chen L. W., Shen G. S. Vibration and buckling of initially stressed curved beams, J. Sound Vib., 215 (3), 511-526, (1998).

17 Nayfeh A. H., Lacarbonara W., and Chin C. H. Nonlinear normal modes of buckled beams: three-to-one and oneto-one internal resonances, Nonlinear Dyn., 18, 253-273, (1999).

18 Tarnopolskaya T., De Hoog F. R., and Fletcher N. H. Lowfrequency mode transition in the free inplane vibration of curved beams, J. Sound Vib., 228 (1), 69-90, (1999).

19 Lestari W. and Hanagud S. Nonlinear vibration of buckled beams: some exact solutions, Int. J. Solids Str., 38, 47414757, (2001). 


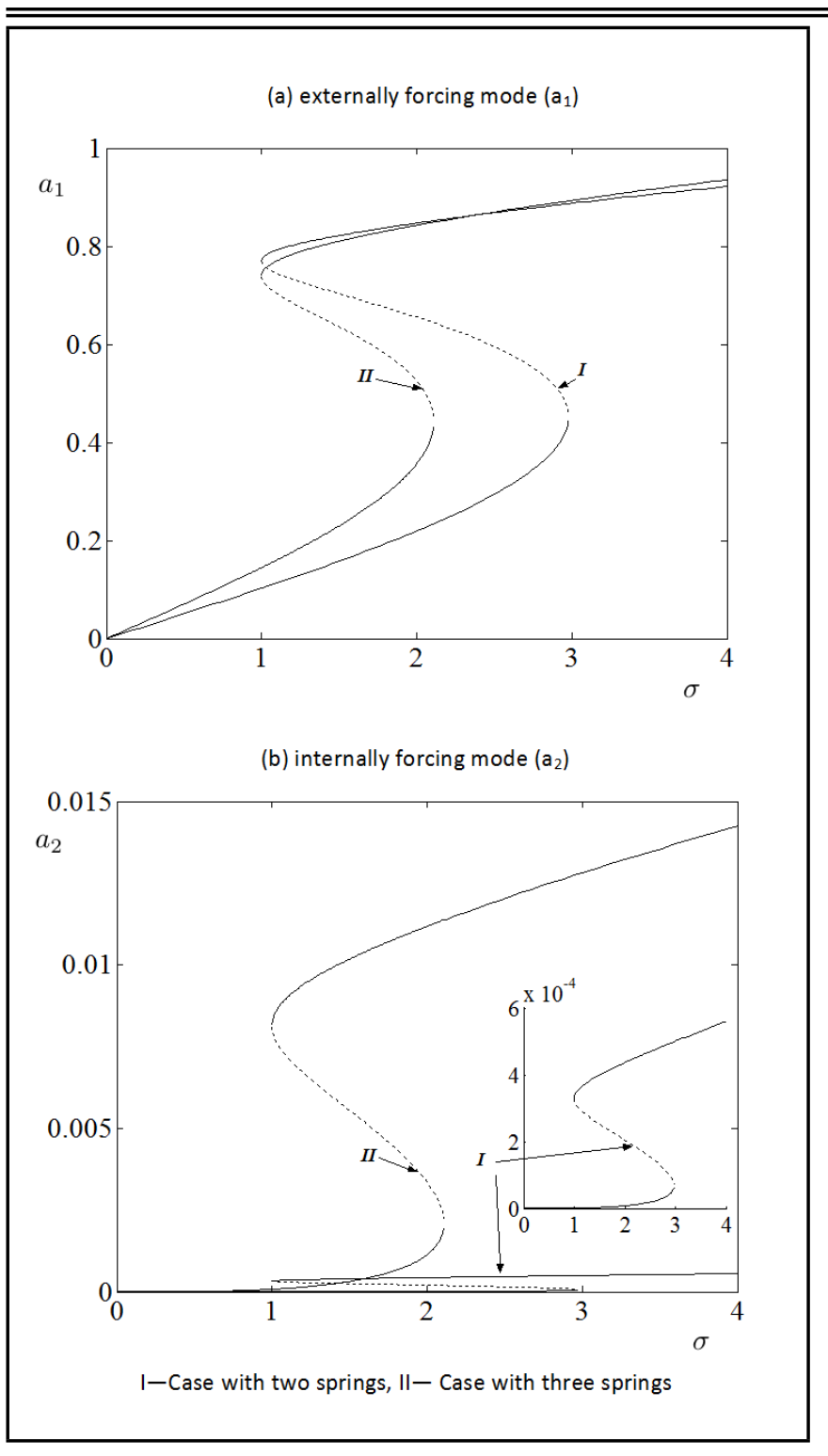

Figure 7. Forcing-response curves.

20 Lacarbonara W., Chin C. M. and Soper R. R. Open-loop nonlinear vibration control of shallow arches via Perturbation approach, J. Appl. Mech, 69, 325-334, (2002).

21 Lacarbonara W. and Rega G. Resonant non-linear normal modes. Part II: activation=orthogonality conditions for shallow structural systems, Int. J. of Non-Linear Mech., 38, 873-887, (2003).

22 Wu J. S. and Chiang L. K. A new approach for free vibration analysis of arches with effects of shear deformation and rotary inertia considered, J. Sound Vib., 277, 49-71, (2004).

23 Adessi D., Lacarbonara W., and Paolone A. Free in-plane vibrations of highly buckled beams carrying a lumped mass, Acta Mech., (2005). http://dx.doi.org/10.1007/s00707-0050259-6

24 Lacarbonara W., Arafat H. N., and Nayfeh A. H. Nonlinear interactions in imperfect beams at veering, Non-Linear Mech., 40, 987-1003, (2005).
25 Ecsedi I. and Dluhi K. A linear model for the static and dynamic analysis of non- homogeneous curved beams, Applied Mathematical Modelling, 29, 1211-1231, (2005).

26 Lee Y. Y., Poon W. Y. and Ng C. F. Anti-symmetric mode vibration of a curved beam subject to auto parametric excitation, J. Sound Vib., 290, 48-64, (2006).

27 Oz H. R. and Das M. T. In-plane vibrations of circular curved beams with a transverse open crack, Math. Comput. Appl., 11 (1), 1-10, (2006).

28 Oz H. R. and Pakdemirli M. Two-to-one internal resonances in a shallow curved beam resting on an elastic foundation, Acta Mech., 185, 245-260, (2006).

29 Ozkaya E., Sarigul M., and Boyaci H. Nonlinear transverse vibrations of a slightly curved beam carrying a concentrated mass, Acta Mech. Sinica, 25 (6), 871-882, (2009).

30 Ozkaya E., Sarigul M. and Boyaci H., Nonlinear transverse vibrations of a slightly curved beam carrying multiple concentrated masses: primary resonance, 2nd International Symposium on Sustainable Development, Sarajevo, Bosnia and Herzegovina, (2010).

31 Xiuchang H., Hongxing H., Yu W., and Zhipeng D. Research on wave mode conversion of curved beam structures by the Wave approach, J. Vib. Acoust., 135 (3), (2013). http://dx.doi.org/10.1115/1.4023817

32 Stancioiu D., Ouyang H., Mottershead J. E. Vibration of a continuous beam with multiple elastic supports excited by a moving two-axle system with separation, Meccanica, 44, 293-303, (2009).

33 Motaghian S. E., Mofid M., and Alanjari P. Exact solution to free vibration of beams partially supported by an elastic foundation, Scientia Iranica A., 18 (4), 861-866, (2011).

34 Motaghian S., Mofid M., and Akin J. E. On the free vibration response of rectangular plates, partially supported on elastic foundation, Applied Mathematical Modeling, 36, 4473-4482, (2012).

35 Ghayesh M. H. Nonlinear dynamic response of a simply supported Kelvin-Voigt viscoelastic beam, additionally supported by a nonlinear spring, Nonlinear Anal. R. World Appl., 13, 1319-1333, (2012).

36 Sari G. and Pakdemirli M. Vibrations of a slightly curved microbeam resting on an elastic foundation with nonideal boundary conditions, Math. Probl. Eng., (2013). http://dx.doi.org/10.1155/2013/736148

37 Nayfeh A.H. and Nayfeh J.F., and Mook D.T. On methods for continuous systems with quadratic and cubic nonlinearities, Nonlinear Dyn., 3, 145-162, (1979).

38 Nayfeh A. H. Introduction to perturbation techniques, Willey, New York, (1981). 


\section{APPENDIX}

$$
\begin{aligned}
& \frac{d}{d t}=D_{0}+\varepsilon \cdot D_{1}+\varepsilon^{2} \cdot D_{2}+\ldots, \frac{d^{2}}{d t^{2}}=D_{0}^{2}+2 \cdot \varepsilon \cdot D_{0} \cdot D_{1}+\varepsilon^{2} \cdot\left(D_{1}^{2}+2 \cdot D_{0} \cdot D_{2}\right)+\ldots \\
& \Gamma_{p+1}=\sum_{r=0}^{n} \int_{\eta_{r}}^{\eta_{r+1}}\left\langle Y_{r+1}^{\prime} \cdot \varphi_{(r+1) 1}^{\prime}+2 \cdot Y_{r+1}^{\prime} \cdot \varphi_{(r+1) 2}^{\prime}\right\rangle d x \cdot Y_{0}^{\prime \prime} \\
& +\sum_{r=0}^{n} \int_{\eta_{r}}^{\eta_{r+1}}\left\langle\frac{3}{2} \cdot Y_{r+1}^{\prime 2}+Y_{0}^{\prime} \cdot \varphi_{(r+1) 1}^{\prime}+2 \cdot Y_{0}^{\prime} \cdot \varphi_{(r+1) 2}^{\prime}\right\rangle d x \cdot Y_{p+1}^{\prime \prime}+\sum_{r=0}^{n} \int_{\eta_{r}}^{\eta_{r+1}} Y_{0}^{\prime} \cdot Y_{r+1}^{\prime} d x \cdot\left[\varphi^{\prime \prime}{ }_{(p+1) 1}+2 \cdot \varphi^{\prime \prime}{ }_{(p+1) 2}\right] . \\
& \sum_{r=0}^{n} \int_{\eta_{r}}^{\eta_{r+1}} Y_{r+1}^{2} \cdot d x=1, \quad f=\sum_{r=0}^{n} \int_{\eta_{r}}^{\eta_{r+1}} F_{r+1} \cdot Y_{r+1} d x \\
& \gamma=\sigma \cdot T 2-\theta, \quad \lambda=-\frac{1}{8 \cdot \omega} \cdot\left\{\sum_{r=0}^{n} \int_{\eta_{r}}^{\eta_{r+1}} Y_{r+1} \cdot \Gamma_{r+1} d x\right\} \\
& \Gamma_{(p+1) 1}{ }^{(k)}=\sum_{r=0}^{n} \int_{\eta_{r}}^{\eta_{r+1}}\left\langle Y_{(r+1) k}^{\prime} \cdot \varphi^{\prime}{ }_{(r+1) 1}+2 \cdot Y_{(r+1) k}^{\prime} \cdot \varphi^{\prime}{ }_{(r+1) 5}\right\rangle d x \cdot Y_{0}^{\prime \prime} \\
& +\sum_{r=0}^{n} \int_{\eta_{r}}^{\eta_{r+1}} Y_{0}^{\prime} \cdot Y_{(r+1) k}^{\prime} d x \cdot\left[\varphi^{\prime \prime}{ }_{(p+1) 1}+2 \cdot \varphi^{\prime \prime}{ }_{(p+1) 5}\right] \\
& +\sum_{r=0}^{n} \int_{\eta_{r}}^{\eta_{r+1}}\left\langle Y^{\prime}{ }_{0} \cdot \varphi^{\prime}{ }_{(r+1) 1}+2 \cdot Y^{\prime}{ }_{0} \cdot \varphi^{\prime}{ }_{(r+1) 5}+\frac{3}{2} \cdot Y_{(r+1) k}^{\prime 2}\right\rangle d x \cdot Y^{\prime \prime}{ }_{(p+1) k} \\
& \Gamma_{(p+1) 2}{ }^{(k)}=\sum_{r=0}^{n} \cdot \int_{\eta_{r}}^{\eta_{r+1}}\left\langle 2 \cdot Y_{(r+1) k}^{\prime} \cdot \varphi^{\prime}{ }_{(r+1) 6}+Y_{(r+1) s}^{\prime} \cdot \varphi_{(r+1) 3}^{\prime}+Y_{(r+1) s}^{\prime} \cdot \varphi^{\prime}(r+1) 4\right\rangle d x \cdot Y_{0}^{\prime \prime} \\
& +2 \cdot \sum_{r=0}^{n} \int_{\eta_{r}}^{\eta_{r+1}} Y_{0}^{\prime} \cdot Y^{\prime}{ }_{(r+1) k} d x \cdot \varphi^{\prime \prime}{ }_{(p+1) 6}+\sum_{r=0}^{n} \int_{\eta_{r}}^{\eta_{r+1}}\left\langle Y_{(r+1) s}^{\prime 2}+2 \cdot Y_{0}^{\prime} \cdot \varphi^{\prime}{ }_{(r+1) 6}\right\rangle d x \cdot Y^{\prime \prime}{ }_{(p+1) k} \\
& +\sum_{r=0}^{n} \int_{\eta_{r}}^{\eta_{r+1}} Y_{0}^{\prime} \cdot Y_{(r+1) s}^{\prime} d x \cdot\left[\varphi^{\prime \prime}{ }_{(p+1) 3}+\varphi^{\prime \prime}{ }_{(p+1) 4}\right] \\
& +\sum_{r=0}^{n} \int_{\eta_{r}}^{\eta_{r+1}}\left\langle Y_{0}^{\prime} \cdot \varphi^{\prime}{ }_{(r+1) 3}+Y_{0}^{\prime} \cdot \varphi^{\prime}{ }_{(r+1) 4}+2 \cdot Y_{(r+1) k}^{\prime} \cdot Y_{(r+1) s}^{\prime}\right\rangle d x \cdot Y_{(p+1) s}^{\prime \prime} \\
& \Gamma_{(p+1) 3}{ }^{(k)}=\sum_{r=0}^{n} \int_{\eta_{r}}^{\eta_{r+1}}\left\langle Y_{(r+1) k}^{\prime} \cdot \varphi_{(r+1) 4}^{\prime}+Y_{(r+1) s}^{\prime} \cdot \varphi_{(r+1) 1}^{\prime}\right\rangle d x \cdot Y_{0}^{\prime \prime} \\
& +\sum_{r=0}^{n} \int_{\eta_{r}}^{\eta_{r+1}}\left\langle Y_{0}^{\prime} \cdot \varphi^{\prime}{ }_{(r+1) 4}+Y_{(r+1) k}^{\prime} \cdot Y_{(r+1) s}^{\prime}\right\rangle d x \cdot Y^{\prime \prime}{ }_{(p+1) k}+\sum_{r=0}^{n} \int_{\eta_{r}}^{\eta_{r+1}}\left\langle Y_{0}^{\prime} \cdot \varphi^{\prime}{ }_{(r+1) 1}+\frac{1}{2} \cdot Y_{(r+1) k}^{\prime 2}\right\rangle d x \cdot Y^{\prime \prime}{ }_{(p+1) s} \\
& +\sum_{r=0}^{n} \int_{\eta_{r}}^{\eta_{r+1}} Y_{0}^{\prime} \cdot Y^{\prime}{ }_{(r+1) k} d x \cdot \varphi^{\prime \prime}{ }_{(p+1) 4}+\sum_{r=0}^{n} \int_{\eta_{r}}^{\eta_{r+1}} Y^{\prime}{ }_{0} \cdot Y^{\prime}{ }_{(r+1) s} d x \cdot \varphi^{\prime \prime}{ }_{(p+1) 1}
\end{aligned}
$$




$$
\begin{aligned}
& \Gamma_{(p+1) 1}{ }^{(s)}=\sum_{r=0}^{n} \int_{\eta_{r}}^{\eta_{r+1}}\left\langle Y^{\prime}{ }_{(r+1) s} \cdot \varphi^{\prime}{ }_{(r+1) 2}+2 \cdot Y^{\prime}{ }_{(r+1) s} \cdot \varphi^{\prime}{ }_{(r+1) 6}\right\rangle d x \cdot Y^{\prime \prime}{ }_{0} \\
& +\sum_{r=0}^{n} \int_{\eta_{r}}^{\eta_{r+1}}\left\langle Y_{0}^{\prime} \cdot \varphi_{(r+1) 2}^{\prime}+2 \cdot Y_{0}^{\prime} \cdot \varphi_{(r+1) 6}^{\prime}+\frac{3}{2} \cdot Y_{(r+1) s}^{\prime 2}\right\rangle d x \cdot Y_{(p+1) s}^{\prime \prime} \\
& +\sum_{r=0}^{n} \int_{\eta_{r}}^{\eta_{r+1}} Y_{0}^{\prime} \cdot Y^{\prime}{ }_{(r+1) s} d x \cdot\left[\varphi^{\prime \prime}{ }_{(p+1) 2}+2 \cdot \varphi^{\prime \prime}{ }_{(p+1) 6}\right] \\
& \Gamma_{(p+1) 2}{ }^{(s)}=\sum_{r=0}^{n} \int_{\eta_{r}}^{\eta_{r+1}}\left\langle Y_{(r+1) k}^{\prime} \cdot \varphi_{(r+1) 3}^{\prime}+Y_{(r+1) k}^{\prime} \cdot \varphi_{(r+1) 4}^{\prime}+2 \cdot Y_{(r+1) s}^{\prime} \cdot \varphi_{(r+1) 5}^{\prime}\right\rangle d x \cdot Y^{\prime \prime}{ }_{0} \\
& +2 \cdot \sum_{r=0}^{n} \int_{\eta_{r}}^{\eta_{r+1}} Y_{0}^{\prime} \cdot Y^{\prime}{ }_{(r+1) s} d x \cdot \varphi^{\prime \prime}{ }_{(p+1) 5}+\sum_{r=0}^{n} \int_{\eta_{r}}^{\eta_{r+1}}\left\langle Y_{0}^{\prime} \cdot \varphi_{(r+1) 3}^{\prime}+Y_{0}^{\prime} \cdot \varphi_{(r+1) 4}^{\prime}+2 \cdot Y_{(r+1) k}^{\prime} \cdot Y_{(r+1) s}^{\prime}\right\rangle d x \cdot Y^{\prime \prime}{ }_{(p+1) k} \\
& +\sum_{r=0}^{n} \int_{\eta_{r}}^{\eta_{r+1}} Y_{0}^{\prime} \cdot Y^{\prime}{ }_{(r+1) k} d x \cdot\left[\varphi^{\prime \prime}{ }_{(p+1) 3}+\varphi^{\prime \prime}{ }_{(p+1) 4}\right]+\sum_{r=0}^{n} \int_{\eta_{r}}^{\eta_{r+1}}\left\langle 2 \cdot Y_{0}^{\prime} \cdot \varphi_{(r+1) 5}^{\prime}+Y_{(r+1) k}^{\prime 2}\right\rangle d x \cdot Y^{\prime \prime}{ }_{(p+1) s} \\
& \Gamma_{(p+1) 3}{ }^{(s)}=\sum_{r=0}^{n} \int_{\eta_{r}}^{\eta_{r+1}} Y^{\prime}{ }_{(r+1) k} \cdot \varphi_{(r+1) 1}^{\prime} d x \cdot Y^{\prime \prime}{ }_{0}+\sum_{r=0}^{n} \int_{\eta_{r}}^{\eta_{r+1}}\left\langle Y_{0}^{\prime} \cdot \varphi^{\prime}{ }_{(r+1) 1}+\frac{1}{2} \cdot Y_{(r+1) k}^{\prime 2}\right\rangle d x \cdot Y^{\prime \prime}{ }_{(p+1) k} \\
& +\sum_{r=0}^{n} \int_{\eta_{r}}^{\eta_{r+1}} Y_{0}^{\prime} \cdot Y_{(r+1) k}^{\prime} d x \cdot \varphi_{(p+1) 1}^{\prime \prime} \\
& \sum_{r=0}^{n} \int_{\eta_{r}}^{\eta_{r+1}} Y_{(r+1) k}{ }^{2} d x=1, \quad \sum_{r=0}^{n} \int_{\eta_{r}}^{\eta_{r+1}} Y_{(r+1) s}{ }^{2} d x=1, \quad f=\sum_{r=0}^{n} \int_{\eta_{r}}^{\eta_{r+1}} F_{r+1} \cdot Y_{(r+1) k} d x, \\
& \lambda_{c}{ }^{(k)}=\sum_{r=0}^{n} \int_{\eta_{r}}^{\eta_{r+1}} Y_{(r+1) k} \cdot \Gamma_{(r+1) c}{ }^{(k)} d x, \quad \lambda_{c}{ }^{(s)}=\sum_{r=0}^{n} \int_{\eta_{r}}^{\eta_{r+1}} Y_{(r+1) s} \cdot \Gamma_{(r+1) c}{ }^{(s)} d x, \quad c=1,2,3 . \\
& \gamma=\sigma \cdot T_{2}-\theta_{k}, \quad \beta=\theta_{s}-3 \cdot \theta_{k}+q \cdot T_{2}
\end{aligned}
$$

